\title{
Évaluation du risque de liquéfaction des sols sur le site du nouveau port de Tanger
}

B. DEMAY

M.-L. SARGENTONI

SAIPEM-SA

Immeuble Énergies

1 à 7 Av. San Fernando

Montigny-le-Bretonneux

78884 Saint-Quentin-

en-Yvelines cedex

bruno.demay@saipem-sa.com
1. L La présente communication a pour objectif d'exposer l'ensemble des démarches effectuées pour l'analyse du risque de liquéfaction des sols sur le site du nouveau port de Tanger-Méditerranée (Maroc).

L'intérêt de l'étude réside en une analyse exhaustive du risque de liquéfaction des terrains sous-jacents aux caissons de la digue principale. Elle s'est en effet appuyée sur plusieurs phases successives :

- essais in situ (CPT et SPT) :

- essais de laboratoire

- étude de l'influence du chargement apporté par les caissons :

- analyse de stabilité pseudo-statique avec prise en compte de l'accroissement de pression interstitielle :

- étude dynamique d'interaction sol-structure.

Ces différentes phases ont permis d'éclairer les résultats ambigus, voire contradictoires, obtenus dans la phase initiale des essais in situ, phase à laquelle les études de liquéfaction des sols sont trop souvent réduites.

A chaque étape de l'étude des observations intéressantes ont pu être faites, par exemple quant à la plus grande précision des essais CPT vis-à-vis de la préhension du risque de liquéfaction comparés aux essais SPT, à l'apport indispensable des essais de laboratoire dans une telle étude, aux effets du chargement apporté par l'ouvrage, ou encore aux effets d'une liquéfaction partielle quant à la stabilité des caissons de la digue. On a également mis en relief la complexité et les limites d'une étude dynamique d'interaction sol-structure.

Mots-clés ; liquéfaction, caisson, digue, pénétromètre, interaction sol-structure, sable, résistance au cisaillement.

\section{Assessment of soils liquefaction hazard on the new Tangiers harbour site}

The present communication deals with the assessment of solls liquefaction hazard on the site of the new Tangiers harbour in Morocco.

The interest of this study lies in an exhaustive analysis of the liquefaction hazard for the soils that underije the big caissons of the main breakwater. The study was divided in several stages:

- in situ tests (CPT and SPT) ;

- laboratory tests :

- an analysis of the influence of the load due to caissons ;

- a pseudo-static stability analysis, accounting for the increase of pore pressure :

- a dynamic soil-structure interaction analysis.

These successive stages allowed us to clarify the ambiguous or even contradictory results of the preliminary analysis, based on the in-situ tests to which liquefaction assessment studies are too often limited. Several interesting observations have been made, for instance about the greater precision of the CPT tests in liquefaction hazard assessment compared to SPT, about the essential contribution of laboratory tests in such a study, about the effects of the load due to the caissons, or about the effects of a partial liquefaction on the caissons stability. The complexity and the limits of a dynamic soilstructure interaction analysis have also been enlightened.
Key words : liquefaction hazard, breakwater, caisson, CPT, SPT, soil-structure interaction, sand, shear resistance. 


\section{Présentation du projet du nouveau port Tanger-Méditerranée}

\section{1}

\section{Situation géographique}

Le site choisi pour le projet du nouveau port de Tanger-Méditerranée est situé sur le détroit de Gibraltar à $35 \mathrm{~km}$ à l'est de Tanger et à $15 \mathrm{~km}$, à vol d'oiseau, à l'ouest de Ceuta, au lieu dit «Punta Ciress ». Il se présente sous la forme d'une plage de $2 \mathrm{~km}$ de long orientée nord-est/sud-ouest, bordée à l'est par la colline du Djebel At-Tala culminant à $228 \mathrm{~m}$ d'altitude, et à l'ouest par le relief intitulé Dhat Al Haq.

\section{2}

\section{Les intervenants}

Le maître d'ouvrage de l'opération représentant l'État marocain est l'Agence spéciale Tanger-Méditerranée (TMSA). Le maître d'œuvre délégué est la DAPTM représentant le ministère de l'Équipement et du Transport. Ces entités sont assistées dans leur tâche par les bureaux d'études Ingema et Halcrow. Pour l'évaluation du risque de liquéfaction des sols TMSA s'est attaché les services du professeur Leslie T. Youd.

Les travaux de réalisation du port (lots 1 et 2) ont été confiés à un groupernent d'entreprises composé de Saipem-SA et du groupe Bouygues, réunis pour la circonstance dans la Société pour la réalisation du nouveau port de Tanger-Méditerranée (SRPTM), laquelle s'est adjoint les services de Mécasol.

\section{Description des ouvrages à réaliser}

Le projet se compose d'une digue principale d'une longueur de $2056 \mathrm{~m}$, composée pour une partie d'une digue à talus traditionnelle et pour une autre partie d'une digue à caissons préfabriqués, et d'une digue secondaire de $588 \mathrm{~m}$, encadrant une zone où un tirant d'eau minimal est requis, faisant l'objet de dragages.

Les caissons sont d'abord préfabriqués en partie à terre dans une zone remblayée conquise sur la mer, puis mis à l'eau en flottaison et rehaussés jusqu'à la hauteur requise en étant amarrés à un quai de rehausse. Ils sont ensuite remorqués à l'endroit de leur échouage puis finalement ballastés par un remplissage de sable issu du dragage (Fig. 1). L'assise des caissons est à la côte -20 et ceux-ci reposent sur un tout-venant $0 / 500 \mathrm{~kg}$ réglé à la côte requise par du ballast, ces matériaux étant clapés sur le fond marin préalablement à l'échouage des caissons. L'emprise au sol des caissons est de l'ordre de $28 \mathrm{~m} \times 28 \mathrm{~m}$ et ils apportent sur le sol une surcharge de $300 \mathrm{kPa}$. Leur hauteur est d'environ $35 \mathrm{~m}$.

\section{2}

\section{Contexte géologique}

Les terrains rencontrés à l'emplacement du site du nouveau port de Tanger-Méditerranée sont constitués d'une couverture quaternaire reposant sur un socle tertiaire et secondaire appartenant aux nappes de flysch de Tisirène et de Beni Ider.

Les dépôts quaternaires ont une origine fluviatile et marine:

- sédimentation marine: sable fin à moyen coquillier recouvrant les formations hétérogènes fluviatiles :

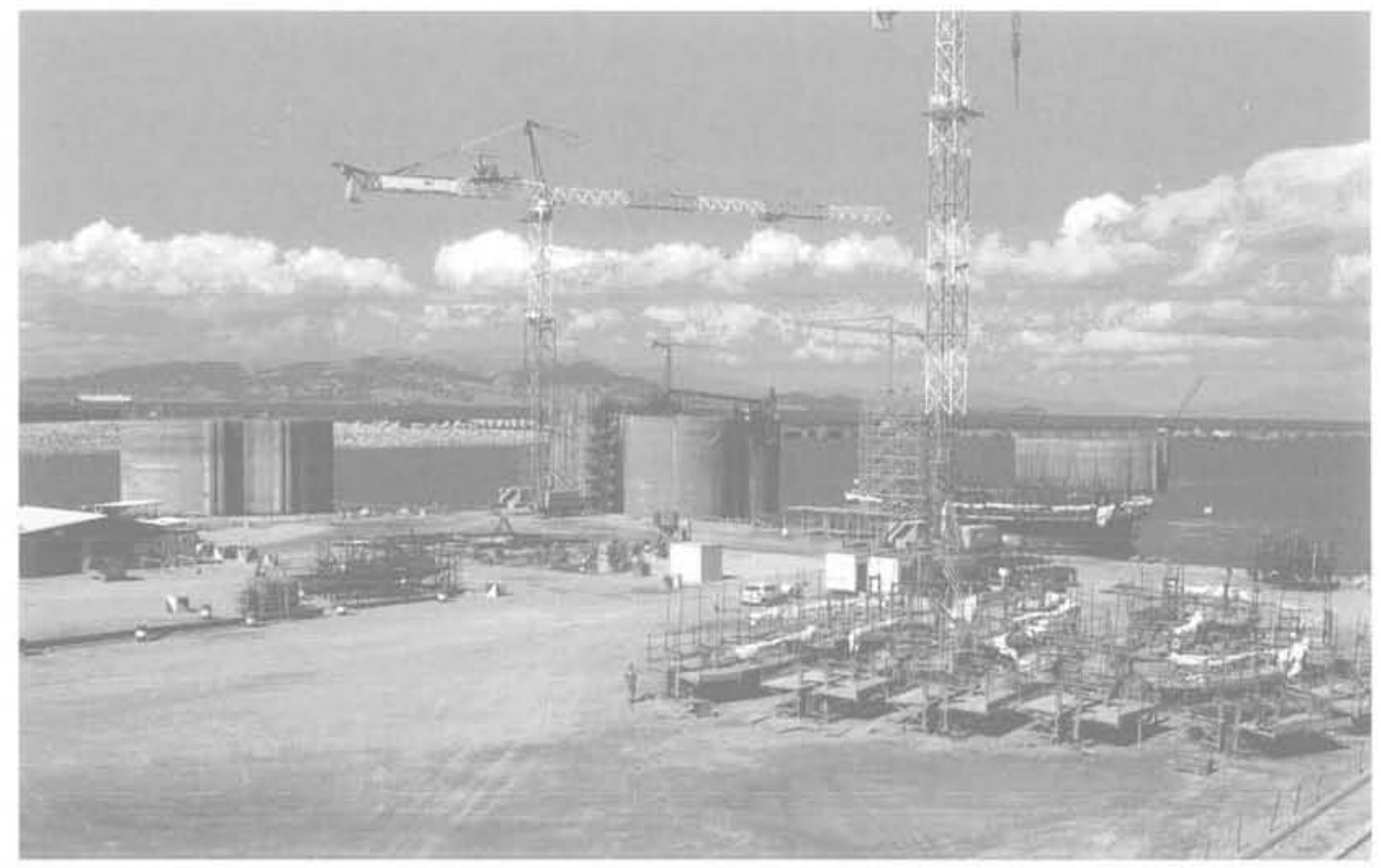

FG 1 Le chantier en octobre 2005 : caissons en cours de fabrication. A l'arrière-plan, la digue en caissons. Works in October, 2005: Caissons at precasting yard. See the caissons breakwater in the background. 
- sédimentation fluviatile due à l'oued R'Mel : alternances de sable, d'argile et graviers très hétérogènes, d'argile molle avec un faible taux de matières organiques, de sables grossiers et très fins, de graves.

D'après la carte géologique (feuille de Sebta/Nord du Maroc) les terrains composant le substratum tertiaire et secondaire de la vallée de l'oued $\mathrm{R}^{\prime} \mathrm{Mel}$ et les collines bordant le site du port sont constitués :

- à l'ouest, d'alternances de grès micacés calcareux et de pélites (Oligocène, nappe du flysch des Beni Ider) ;

- au centre, de calcaires, de calcaires et marnes micacées rouges (Paléocène-Eocène), puis de marno-calcaires (Crétacé supérieur, BenI Ider)

- à l'est, d'une alternance de grès vert jaune à grain fin et de pélites (Crétacé inférieur, nappe du flysch du Djebel Tisirène). En particulier, cette formation concerne la colline du Djebel At-Tala qui domine l'est du site.

La profondeur du fond marin varie de 0 à $-35 \mathrm{~m}$ selon les cartes bathymétriques existantes, avec une pente relativement régulière de 3 à $4 \%$ en direction du nord-ouest.

La profondeur d'eau maximale est de 35 mètres dans la zone située à l'extérieur du musoir de la digue principale (Fig. 2).

\section{3}

\section{Conditions sismiques}

Le maître d'ouvrage TMSA a chargé J'université Mohammed V de Rabat de définir l'aléa sismique sur le site du futur port. Ces données ont été explicitement intégrées aux conditions techniques du contrat de réalisation.
Selon les propres termes du rapport établi par l'université, la démarche suivie par les spécialistes marocains pour l'élaboration de ces données a consisté en - une synthèse de l'évaluation de l'aléa sismique sur le site du futur port par le biais d'un recueil et d'une analyse des principaux documents géologiques (analyse structurale/néotectonique/stratigraphie), géophysiques et sismologiques disponibles à ce jour ;

- une analyse critique et une synthèse des données de base recueillies depuis l'échelle du territoire du Maroc jusqu'à l'échelle du site ayant pour objet un zonage sismotectonique définissant les zones sources et les principales failles et séismes associés pouvant avoir une influence sur le site du futur port;

- une sélection des séismes majeurs des zones sources (zones sismotectoniques et failles) en fonction de l'état des connaissances et des données disponibles, afin d'évaluer leur potentialité en terme de magnitudes et d'intensités macrosismiques (échelle MSK 1964) susceptibles de se produire sur le site :

- une définition des caractéristiques (intensité, magnitude, distance, profondeur) des séismes de référence conformément à la pratique réglementaire pour les installations classées définissant le tremblement de terre de dimensionnement (SMHV) et le séisme majoré de sécurité (SMS) ; - enfin, le calcul les accélérations maximales plausibles. pour chaque séisme de référence, à partir de relations empiriques couramment utilisées.

Selon l'étude conduite par l'université marocaine, il apparait que plusieurs sources sismiques conduisent aux mouvements les plus pénalisants sur le site. Il s'agit de la source locale, qui génère les mouvements les plus pénalisants dans le domaine des hautes fréquences, et les deux sources lointaines du golfe de Cadix et du SW du cap Saint-Vincent qui génèrent les mouvements les plus pénalisants dans le domaine des basses fréquences :

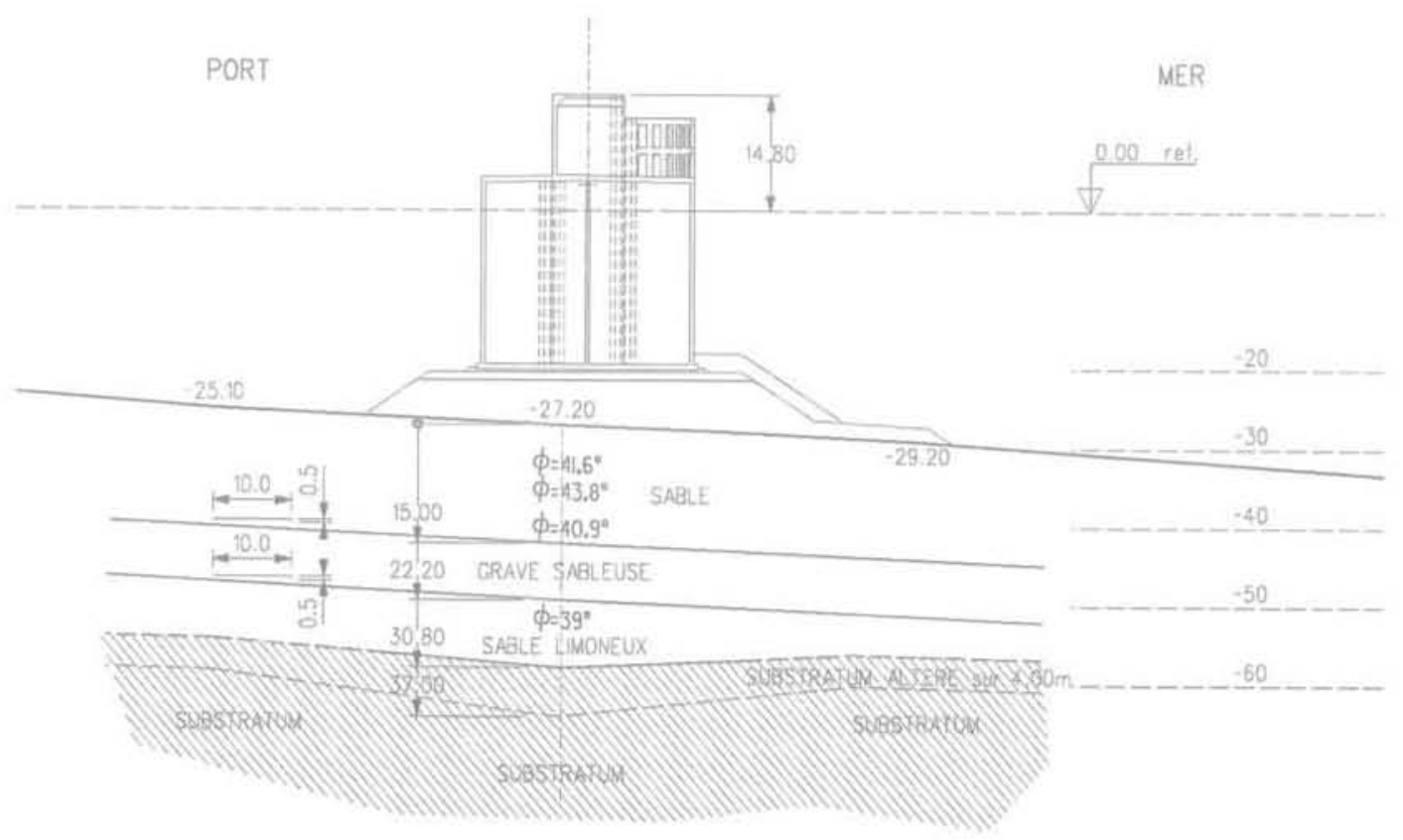

Port de Tanger-Méditerranée : digue principale. Coupe transversale vers le PM 2050.

FG.2 Une coupe type des terrains sous les caissons.

A typical geological cross-section under the caissons. 


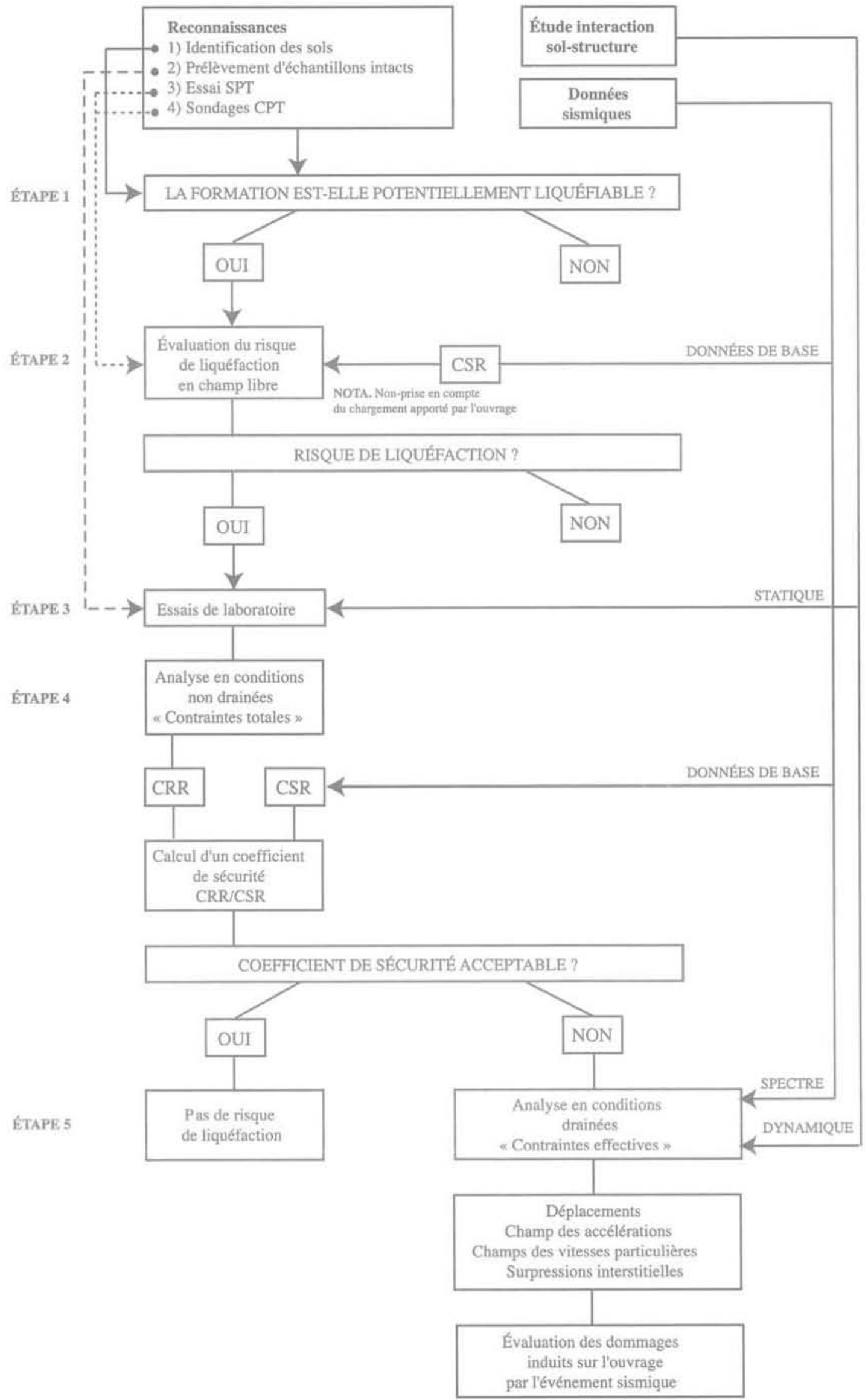

Fig. 3 Méthodologie suivie pour l'étude de la liquéfaction Methodology for liquefaction hazard assessment. 
- la source proche (celle du site), qui conduit aux mouvements les plus pénalisants pour un séisme de type SMS avec une accélération horizontale maximale $\mathrm{a}_{\mathrm{h}}=$ $0,24 \mathrm{~g}$ au site pour les hautes fréquences (magnitude retenue de 4,7 ) :

- la source lointaine (SW du cap Saint-Vincent) qui conduit aux mouvements les plus pénalisants pour un séisme de type SMS avec une accélération horizontale maximale $a_{h}=0,093 \mathrm{~g}$ au site pour les basses fréquences (magnitude retenue de 8,5).

Il est à noter que, dans cette étude, les conditions particulières de site n'ont pas été prises en compte, car les mouvements spécifiés sont fournis pour un site rocheux sans tenir compte des amplifications ou des atténuations potentielles liées à l'influence des terrains sus-jacents. Également, l'étude ne fournit pas de signal sismique ou de spectre de référence pour une éventuelle étude spécialisée.

\section{Méthode développée pour l'analyse du risque de liquéfaction}

\section{1}

\section{Généralités}

Dans ce qui suit, le terme de liquéfaction correspond au phénomène de perte de résistance au cisaillement d'un sable fin saturé, lâche ou dense, qui peut avoir lieu pendant un séisme à cause de l'augmentation des pressions interstitielles.

Les contraintes de cisaillement cycliques dues aux phénomènes vibratoires causés par le séisme entraînent une augmentation de la pression interstitielle, et la théorie veut que lorsque cette pression atteint la valeur de la contrainte totale en place, annulant ainsi la contrainte effective, le sable perd l'essentiel de sa résistance au cisaillement et on dit qu'il a liquéfié.

La méthodologie utilisée dans le cadre de ce projet est résumée sur le logigramme de la figure 3.

Par rapport aux démarches courantes en la matière, la méthodologie proposée faisait explicitement référence à des essais de laboratoire, nécessaires à l'identification des sols et à la mesure de la résistance à la liquéfaction dans des conditions simulant l'état de contraintes du sol en place avant et après construction de l'ouvrage.

Ces essais de laboratoire ont été conduits au NGI (Norwegian Geotechnical Institute) à Oslo et chez Geolabo au Plessis-Robinson.

\section{2}

\section{Critères d'identification des sols potentiellement liquéfiables}

Selon les recommandations AFPS en vigueur sont a priori suspects de liquéfaction les sables, sables vasards et silts présentant les caractéristiques suivantes :

- degré de saturation voisin de $100 \%$;

- granulométrie correspondant à un coefficient d'uniformité $\mathrm{Cu}=\mathrm{d}_{60} / \mathrm{d}_{10}$ inférieur à 15 ;
- diamètre à $50 \% \mathrm{~d}_{50}$ compris entre $50 \mu \mathrm{m}$ et $1,5 \mathrm{~mm}$; - et soumis en l'état final du projet à une contrainte verticale effective inférieure à 200 à $300 \mathrm{kPa}$ (valeur variable selon des zones définies en fonction de l'intensité du phénomène sismique).

Ce dernier point est très important dans le cas présent, puisque la contrainte apportée sur le sol par les caissons est de l'ordre de $300 \mathrm{kPa}$ et donc, si l'on suit cette recommandation sans analyse complémentaire, le sol sous l'ouvrage ne doit pas être considéré comme potentiellement liquéfiable. Hormis le problème posé par la délimitation précise des zones influencées par le chargement, l'action de charger le sol ne suffit pas à éliminer les risques inhérents au phénomène de liquéfaction, comme on le verra ci-après.

Sont également à considérer comme liquéfiables les sols argileux présentant les caractéristiques suivantes : - diamètre à $15 \% \mathrm{~d}_{15}>5 \mu \mathrm{m}$;

- limite de liquidité < $35 \%$;

- teneur en eau supérieure à 0,9 fois la limite de liquidité $W_{L}$ :

- et dont le point représentatif sur le diagramme de plasticité se situe au-dessus de la droite du dit diagramme $I_{p}=0,73\left(W_{L}-20\right)$ pour $W_{L}>30$ et $I_{p}=7,3$ pour $W_{L}<30$.

\section{3}

\section{Évaluation du risque de liquéfaction des terrains liquéfiables}

L'évaluation du risque de liquéfaction est fondé sur la définition d'un facteur de sécurité FS qui est défini comme le rapport entre la résistance cyclique normalisée du matériau, CRR (cyclic resistance ratio), et la sollicitation cyclique normalisée induite par le séisme à la même profondeur, CSR (cyclic stress ratio) : FS = CPR/CSR.

On considère, en général, un coefficient de sécurité nécessaire supérieur ou égal à 1,3 pour s'affranchir du risque de liquéfaction (Pecker, 1986). A l'inverse, un coefficient de sécurité strictement inférieur à 1 traduit généralement un risque important de liquéfaction.

\section{4}

\section{Sollicitations cycliques normalisées (CSR)}

Le CSR est déduit de l'accélération maximale du séisme à la surface du sol, $\mathrm{a}_{\operatorname{mnx}}$, selon la formule suivante:

$$
\operatorname{CSR}=0,65 \mathrm{r}_{\mathrm{d}}\left(\mathrm{a}_{\mathrm{max}} / \mathrm{g}\right)\left(\sigma_{\mathrm{v}} / \sigma_{\mathrm{v}}^{\prime}\right)
$$

où :

$\Gamma_{\mathrm{d}} \quad$ coefficient réducteur de la contrainte en fonction de la profondeur ;

g accélération de la gravité ;

$a_{\max }$ accélération maximale du séisme à la surface dusol;

$\sigma_{\mathrm{v}} \quad$ contrainte verticale totale ;

$\sigma_{v} \quad$ contrainte verticale effective ;

Le coefficient $r_{\text {d }}$ est défini conformément aux recommandations NCEER (Youd et Idriss, 2001). 


\section{Prise en compte des données sismiques}

Une double analyse a été faite en considérant les deux séismes de référence définis dans l'étude de l'aléa sismique (cf. § 4) :

- séisme de source lointaine (cap Saint-Vincent) : magnitude $M_{w}=8,5$, accélération $a_{\max }=0,093 \mathrm{~g}$;

- séisme de source proche (NW Rif) : magnitude $\mathrm{M}_{w}=$ 4,7 , accélération $\mathrm{a}_{\max }=0,24 \mathrm{~g}$.

\section{5}

\section{Conclusions tirées des essais in situ}

Une campagne de reconnaissances géotechniques marines a été réalisée au deuxième semestre 2003 par l'entreprise Seacore. Elle incluait des sondages carottè et la réalisation d'essais in situ CPT et SPT.

Les essais CPT et SPT ont été interprétés selon la procédure décrite dans les recommandations NCEER (Youd et Idriss, 2001). L'interprétation des essais CPT et SPT a été revue par le professeur Youd.

Un exemple d'analyse des résultats CPT (Fig. 4) montre clairement l'existence d'un risque de liquéfaction sous séisme de magnitude 8,5 dans certaines zones de la digue principale, correspondant à des terrains constitués par le dépôt de matériaux alluvionnaires récents apportés par l'oued $\mathrm{R}^{\prime} \mathrm{Mel}$.

\section{TANGER-MEDITERRANEE CPT 22 SEACORE $a_{\max } 0,093 \mathrm{~g} \mathrm{Mw} \quad 8,5$}

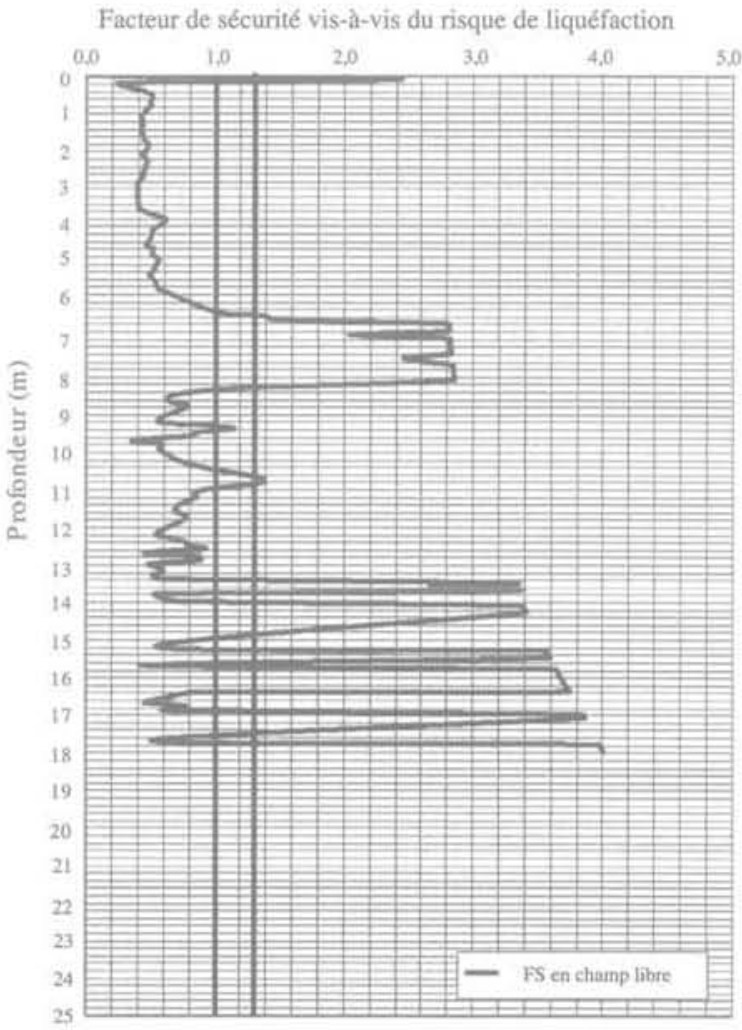

FIG.4 Interprétation d'un essai CPT selon les recommandations NCEER.

CPT interpretation according to NCEEF recommandations.
A contrario, l'interprétation des résultats SPT équivalents (Fig. 5) ne permettait pas de conclure qu'il y avait réellement risque de liquéfaction.

D'une manière générale l'interprétation des essais in situ montrait que le séisme lointain de magnitude 8,5 était plus défavorable vis-à-vis du risque de liquéfaction que le séisme local de magnitude 4,7 , bien que celui-ci soit réputé d'accélération 2,5 fois plus élevée $(0,24 \mathrm{~g}$ contre $0,093 \mathrm{~g})$. Ce résultat est imputable à la prise en compte de l'influence de la magnitude selon les recommandations NCEER.

Il est intéressant de noter que les résultats des sondages SPT étaient en contradiction avec les résultats des essais CPT et concluaient au contraire au fait qu'il n'y avait pas réellement risque de liquéfaction. Il fallait donc lever cette ambiguité.

Par ailleurs, la prise en compte de la surcharge importante apportée par l'ouvrage et de la pente naturelle du sol par l'intermédiaire des facteurs $\mathrm{K}_{\mathrm{o}}$ et $\mathrm{K}_{\mathrm{a}}$ selon les recommandations NCEER avait pour effet de réduire significativement le coefficient de sécurité visà-vis de la liquéfaction obtenu dans l'interprétation des essais in situ, alors qu'une interprétation hâtive des recommandations AFPS donnerait à penser que les sols soumis en l'état final du projet à une contrainte verticale effective supérieure à 200 à $300 \mathrm{kPa}$ ne sont à priori pas suspects de liquéfaction.

Pour pouvoir se prononcer sur le risque de liquéfaction il fallait donc d'une part confirmer la susceptibilité des sols à la liquéfaction par d'autres analyses, et

\section{TANGER-MÉDITERRANÉE SPT 108 SEACORE $a_{\max } 0,093 \mathrm{~g} \mathrm{Mw} \quad 8,5$}

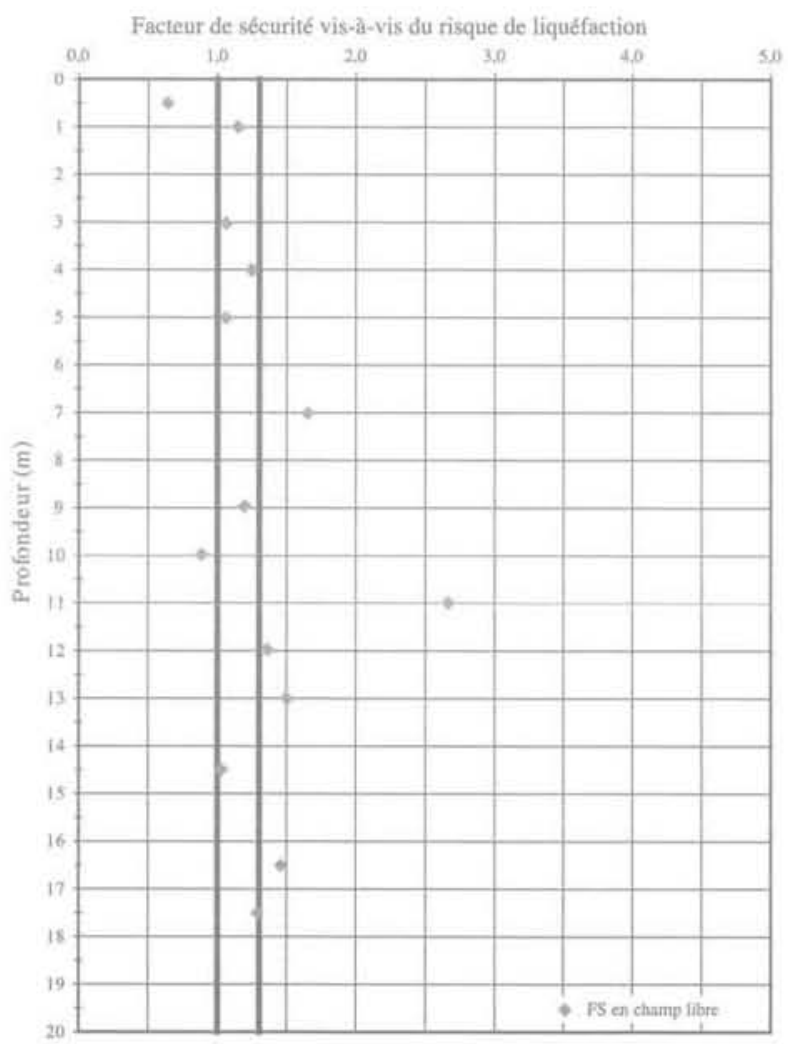

FG.5 Interprétation d'un essai SPT selon les recommandations NCEER. SPT interpretation according to NCEEF recommandations. 
d'autre part estimer le coefficient de sécurité réel en prenant en compte de façon précise l'influence du chargement apporté par l'ouvrage : c'est ici que les essais de laboratoire prennent tout leur sens.

Il fallait également analyser l'incidence de l'amplification ou de l'atténuation du signal sismique à travers les terrains meubles recouvrant le substratum rocheux.

\section{6}

\section{Apport des essais de laboratoire}

\section{1}

\section{Programme d'essais}

La campagne de reconnaissances menée par l'entreprise Seacore a comporté une partie importante de sondages carottés qui ont permis de prélever un certain nombre d'échantillons jugés de qualité suffisante pour y procéder à des essais représentatifs. En particulier, l'utilisation d'un carottier haut de gamme comme le Geobor S Atlas Copco a permis d'avoir des pourcentages de récupération satisfaisants et même dans certains cas l'obtention d'échantillons intacts (Fig, 6).

Les essais de laboratoire ont eu pour but d'apporter des informations sur:

- le caractère potentiellement liquéfiable des sols concernés :

- leur comportement vis-à-vis des sollicitations cycliques à la fois en champ libre et sous un état de contraintes correspondant au chargement apporté par l'ouvrage.

\section{Il a été procédé à :}

- des essais d'identification au sens large (courbes granulométriques, densités mini maxi, limites d'Atterberg...) ;

- des essais de résistance au cisaillement cycliques (essais triaxiaux cycliques, essais à la boite de cisaillement cyclique) visant à évaluer leur résistance au phénomène de liquéfaction ;
- et des essais visant à déterminer le comportement en déformation des sols concernés sous sollicitations cycliques (essais à la colonne résonnante, essais de mesure du module de cisaillement aux petites déformations de cisaillement).

\section{2}

\section{Analyse des essais d'identification}

En ce qui concerne les critères granulométriques relatifs aux sables suspects de liquéfaction, les couches de sols remplissant strictement ce critère de liquéfaction se situaient généralement dans la frange supérieure des sondages carottés. A contrario la partie supérieure d'un sondage, qui était identifié comme très franchement liquéfiable par les CPT voisins, présentait une courbe granulométrique avec un coefficient d'uniformité nettement supérieur à 15 .

Par ailleurs, les formations argileuses rencontrées n'étaient pas dans le cas d'une teneur en eau très proche de la límite de liquidité.

La densité relative des sables, d'après les essais sur les échantillons intacts, s'établissait entre 30 et $70 \%$, ce que confirmait les extrapolations faites à partir des CPT.

\section{3}

\section{Analyse des essais de cisaillement cyclique}

\subsection{1 \\ Généralités}

Il a été effectué une vingtaine d'essais, répartis de façon à peu près équivalente entre l'essai triaxial cyclique et la boite de cisaillement cyclique.

On a cherché à mettre en évidence le caractère déterminant de paramètres susceptibles d'influer sur le déclenchement et l'amplitude du phénomène de liquéfaction :

- les paramètres caractérisant la granulométrie (coefficient d'uniformité, pourcentage de fines) ;

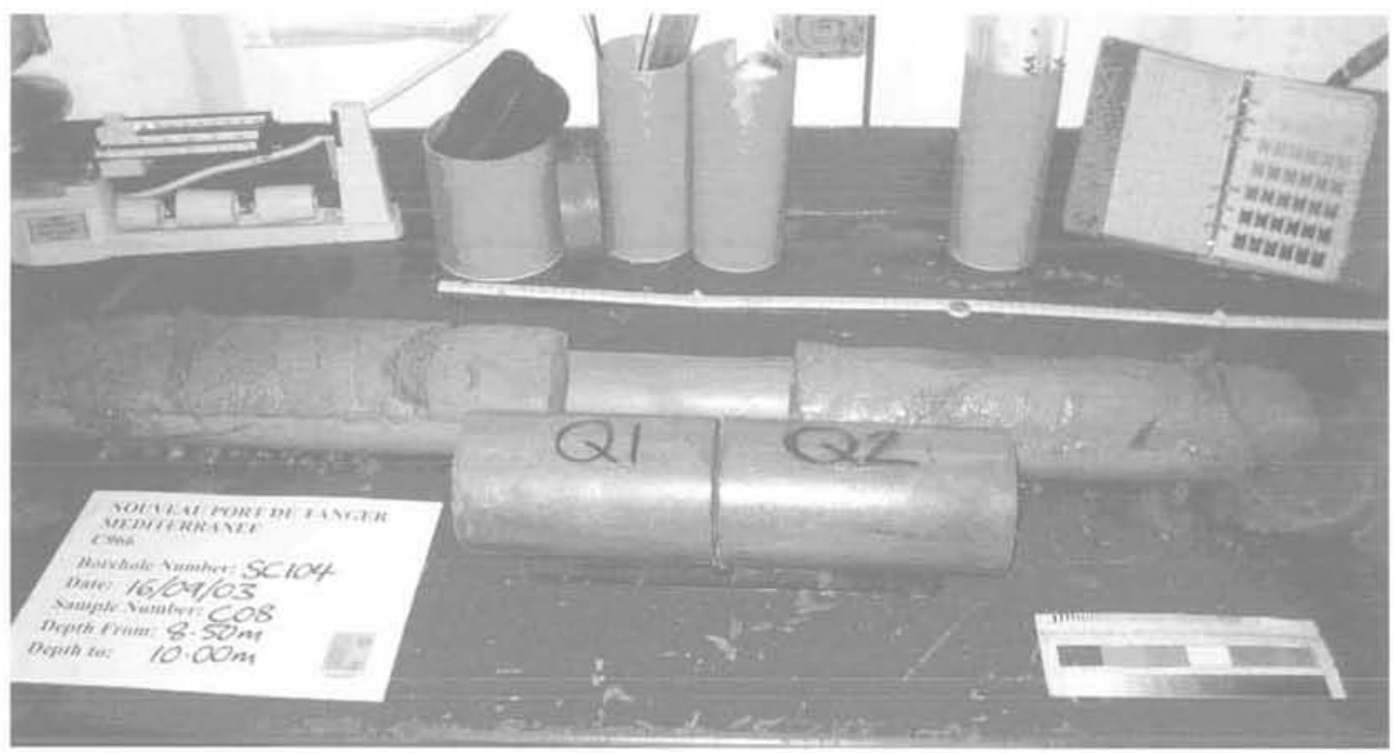

FG. 6 Échantillons intacts dans les sables silteux. Intact samples in loose silty sands. 


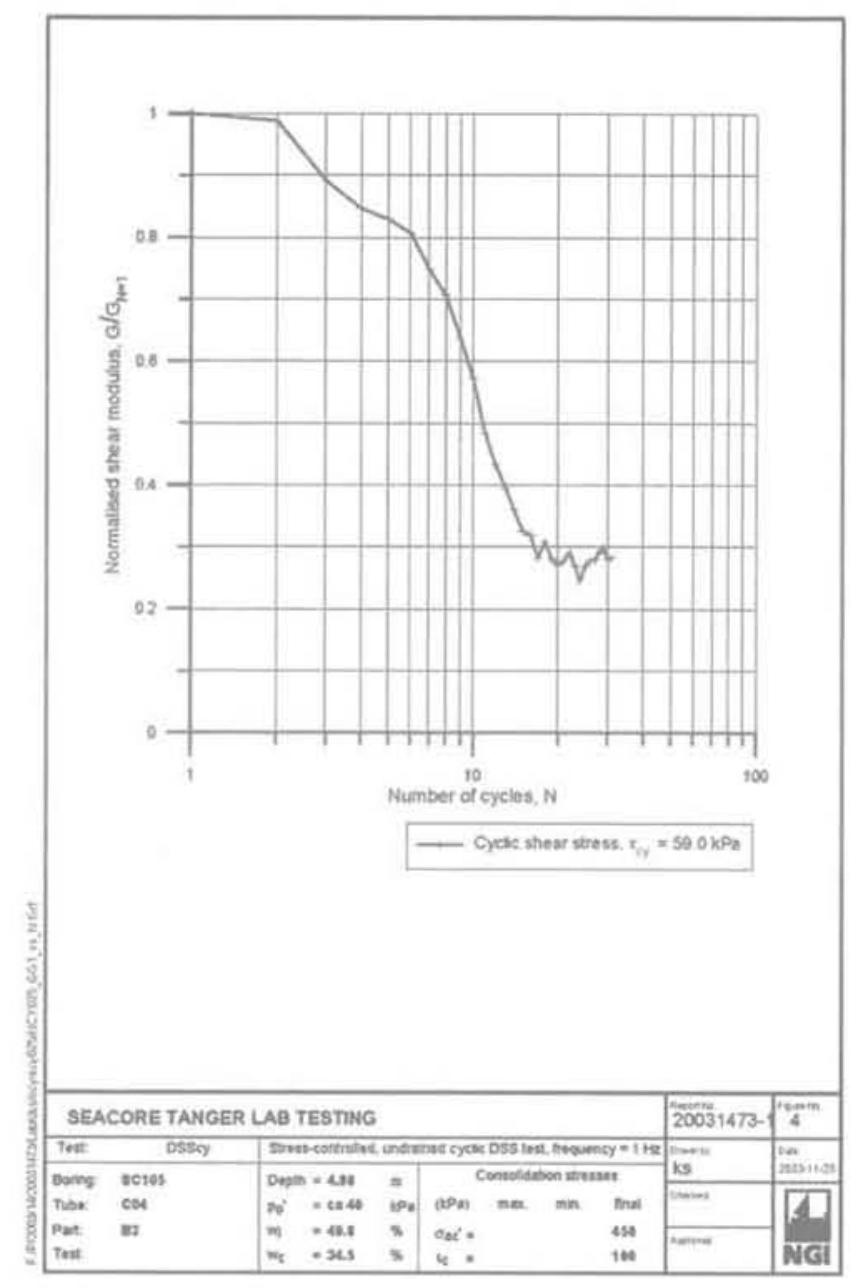

FiG, 7 Évolution du module de cisaillement pendant un essai de cisaillement cyclique. Shear modulus decreases during a direct cyclic shear test.

- la densité relative Dr du sable à l'état initial, c'est-àdire avant consolidation (les échantillons non intacts étaient reconstitués à une valeur de Dr choisie) ;

- la consolidation de l'échantillon, simulant les conditions de chargement (champ libre/chargement apporté par l'ouvrage) ;

- la contrainte de cisaillement statique initiale (avant essai) ;

- l'intensité de la sollicitation cyclique (ici le rapport $\tau / \sigma$ correspondant au CSR selon la formule (1) sera de l'ordre de 0,13 à 0,15 avec une accélération de 0,093 g pour une profondeur comprise entre 0 et $15 \mathrm{~m}$ ).

En fin d'essai, on a noté les valeurs de certains paramètres caractéristiques :

- rapport pression interstitielle/contrainte verticale effective $v / \sigma_{y}^{\prime}$;

- nombre de cycles;

- déformation de cisaillement.

La population des essais se sépare en deux groupes d'égale importance :

- un groupe d'essais réactifs où l'influence des sollicitations cycliques se manifeste par une augmentation significative de la pression interstitielle, une diminution des caractéristiques de déformation (module de

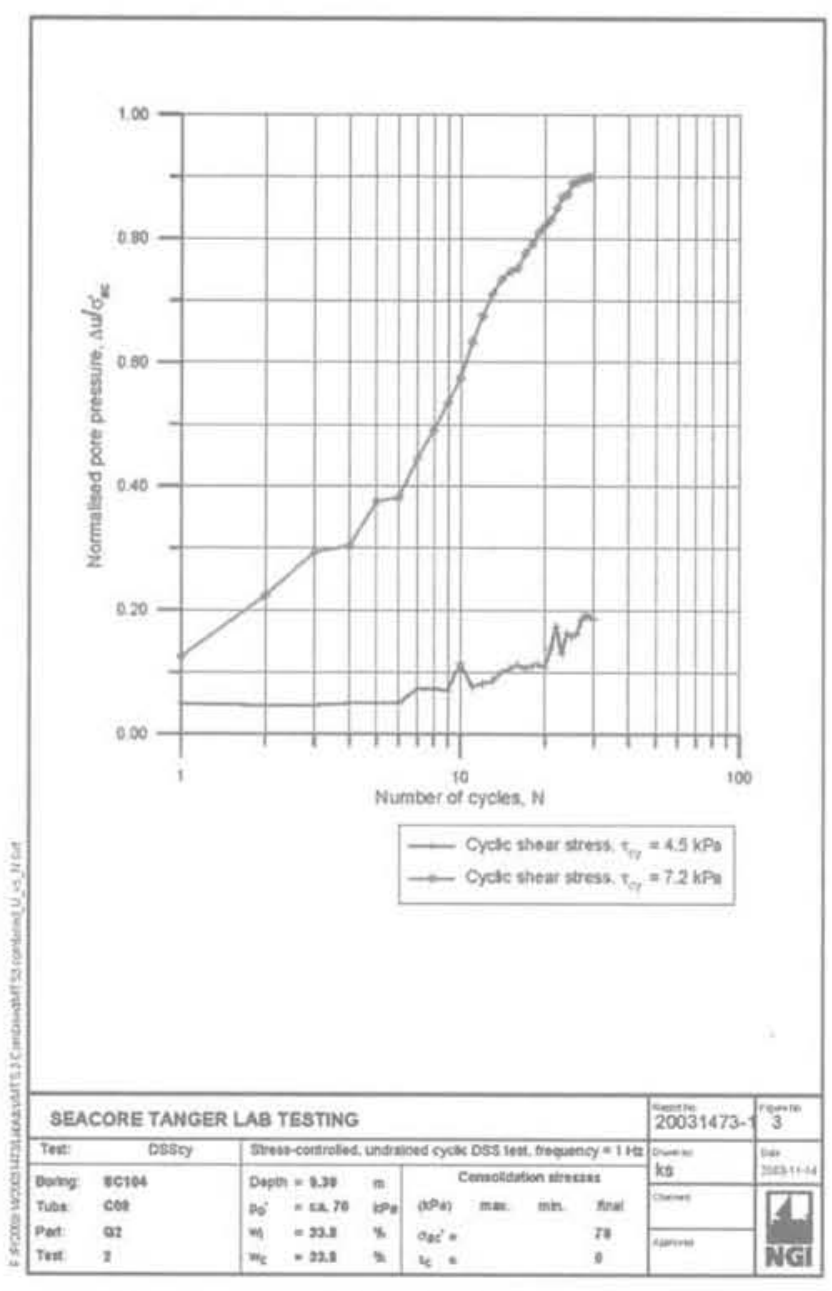

FGG 8 Augmentation de la pression interstitielle Durant un essai de cisaillement cyclique. Pore pressure increases during a direct cyclic shear test.

cisaillement), des déformations de cisaillement finales importantes, le tout pour un nombre de cycles réduit (Fig. 7 et 8);

- un groupe d'essais non réactifs où l'augmentation de pression interstitielle est non significative $\left(\Delta \mathrm{u} / \sigma^{\prime} \mathrm{v}<\right.$ $10 \%$ ) et où les caractéristiques de déformation ne sont pas modifiées.

L'analyse des essais a permis de faire un certain nombre d'observations instructives.

\section{$6,3,2$}

\section{Description des phénomènes observés}

La liquéfaction n'a été réellement observée que sur deux échantillons, soumis à une sollicitation cyclique correspondant à un rapport $\tau / \sigma^{\prime}$ de 0,2 (CSR) ce qui correspondait, toutes choses égales par ailleurs, à une accélération de 0,13 à $0,15 \mathrm{~g}$, soit $50 \%$ de plus que l'hypothèse considérée $(0,093 \mathrm{~g})$.

Les deux essais correspondaient tous deux à une consolidation isotrope, soit à une contrainte de cisaillement initiale nulle. Il est par contre intéressant de noter que l'un correspondait à la situation en champ libre, l'autre à une situation sous chargement. 
Pour un rapport $\tau_{\mathrm{cy}} / \sigma_{\mathrm{v}}^{\prime}$ de l'ordre de 0,13 , correspondant à l'accélération de référence, on a observé un phénomène que nous appellerons liquéfaction partielle, caractérisé par une augmentation de 30 à $60 \%$ de la pression interstitielle et une diminution du module de cisaillement, ainsi que par une valeur importante de la déformation de cisaillement à la fin de l'essai.

Sur les échantillons n'ayant pas liquéfié, les essais de cisaillement jusqu'à rupture post-cycliques montraient un angle de frottement résiduel de l'ordre de $40^{\circ}$, soit la valeur d'origine. Par contre, les courbes efforts/déformations montraient des accroissements de déformation importants pour une faible augmentation des contraintes le long de la surface de rupture, ce qui traduit une diminution significative des modules de cisaillement.

Ces résultats, montrant un état intermédiaire entre la liquéfaction franche et une absence de réaction aux sollicitations cycliques, permettaient d'affiner la perception du risque de liquéfaction que l'on avait après l'interprétation des essais in situ : les essais CPT étaient probablement conservatifs et, inversement, les essais SPT étaient trop optimistes en ce sens qu'ils ne révélaient pas l'amplitude du phénomène affectant les caractéristiques de résistance et de déformation des terrains.

\section{$6.3,3$}

\section{Influence de la densité relative}

Tous les essais réactifs, c'est-à-dire ayant montré un accroissement significatif de la pression interstitielle, correspondaient à des échantillons de densité relative initiale de l'ordre de 40 à $55 \%$.

\section{$6,3.4$}

\section{Influence des caractéristiques granulométriques}

Les deux échantillons ayant liquéfié sont des sables propres ( $7 \%$ de fines) qui possèdent une granulométrie uniforme (coefficient d'uniformité $=3$ ) ; mais on remarque que des échantillons ayant 15 à $20 \%$ de fines et un coefficient d'uniformité de 40 subissent une augmentation importante de la pression interstitielle. Le critère granulométrique de l'AFPS n'est donc pas un critère exclusif.

\section{3 .5}

\section{Conditions de chargement}

Si lion fait référence aux deux essais ayant liquéfié, dans un cas la liquéfaction survient en champ libre, dans l'autre sous chargement. Dans le reste des essais réactifs, il y a autant de situations de chargement que de situations de champ libre.

La seule augmentation de la contrainte verticale sous l'effet du chargement dủ à l'ouvrage n'est donc pas une condition de nature à empêcher la liquéfaction. Le critère de densité relative reste par contre essentiel.

\subsection{6}

\section{Influence de la contrainte de cisaillement statique initiale}

Tous les essais jugés non réactifs sont des essais où la contrainte de cisaillement statique initiale avant essai est non nulle soit par consolidation anisotrope à l'appa- reil triaxial, soit par imposition d'une contrainte de cisaillement préalable à la boîte de cisaillement. Le rapport $\tau_{\mathrm{a}} / \sigma_{\gamma}^{\prime}$ y est supérieur à 0,22 .

A l'inverse, tous les essais qui donnent lieu à un phénomène notable (liquéfaction, augmentation significative de la pression interstitielle, déformation excessive, diminution du module) sont des essais où le cisaillement initial $(\tau)$ peut être estimé nul (ou très faible). Les deux essais qui échapperaient à priori à cette règle sont des essais à la boitte de cisaillement cyclique (DSS), fortement consolidés, où l'on ne connait pas en réalité le niveau de contrainte horizontale s'exerçant sur l'échantillon après consolidation mais où l'on peut supposer qu'il est loin d'être négligeable.

On retrouve ici un résultat mis en évidence dans la littérature (Pecker, 1986 ; Vaid et al., 2001), relatif au rôle joué par la contrainte de cisaillement statique à laquelle vient se superposer à la contrainte de cisaillement cyclique, qui se produit ainsi autour d'une valeur non nulle de cisaillement. Seul le cas où la valeur de la contrainte de cisaillement cyclique est suffisamment élevée pour inverser la direction du cisaillement est susceptible de conduire à la liquéfaction.

Ces résultats conduisaient tout naturellement à examiner de façon détaillée la répartition des contraintes dans le sol sous l'effet du chargement apporté par l'ouvrage, puisqu'il a plusieurs effets concomitants - un tel niveau de charge (300 kPa) augmente nécessairement la compacité des sables sous-jacents et accroît leur densité relative :

- il va générer une contrainte de cisaillement statique qui va se superposer aux sollicitations cycliques sismiques, mais la répartition de cette contrainte de cisaillement varie spatialement sous l'ouvrage.

\section{$=7$ \\ Étude de l'influence du chargement apporté par l'ouvrage}

Nous avons donc étudié à l'aide d'un modèle de calcul aux éléments finis réalisé avec le code PLAXIS l'influence du chargement apporté par l'ouvrage sur la répartition des contraintes dans le sol.

La surcharge importante apportée par le remblai d'assise et les caissons a pour effet de modifier la contrainte verticale mais aussi la contrainte de cisaillement statique initiale.

Le calcul aux éléments finis (en déformation plane) permet de calculer en tout point du maillage la valeur des contraintes verticales et horizontales (soit $\sigma_{y}$ et $\sigma_{x}$ ) ainsi que de la contrainte de cisaillement statique $\tau_{x x^{*}}$. On trace ensuite des coupes parallèles à la surface libre à différentes profondeurs $(1 \mathrm{~m}, 2 \mathrm{~m}, 5 \mathrm{~m}, 10 \mathrm{~m}$...) sur lesquelles on obtient les valeurs de la contrainte verticale effective et de la contrainte de cisaillement statique ; on en déduit alors le rapport des contraintes $\tau_{\mathrm{a}} / \sigma_{\mathrm{V}}^{\prime}$ préexistant à la sollicitation sismique.

Un exemple de courbes donnant la valeur du rapport $\tau / \sigma_{y}^{\prime}$ est donné dans la figure 9 . La courbe part de 0 à l'axe de l'ouvrage, elle passe par un maximum à une distance d de l'axe (ordre de grandeur $d=25 \mathrm{~m}$, variable avec la profondeur) avant de tendre asymptotiquement vers 0 en changeant de sens. La courbe n'est pas symétrique par rapport à l'axe des caissons, compte tenu de la géométrie de l'ouvrage et de la pente. 


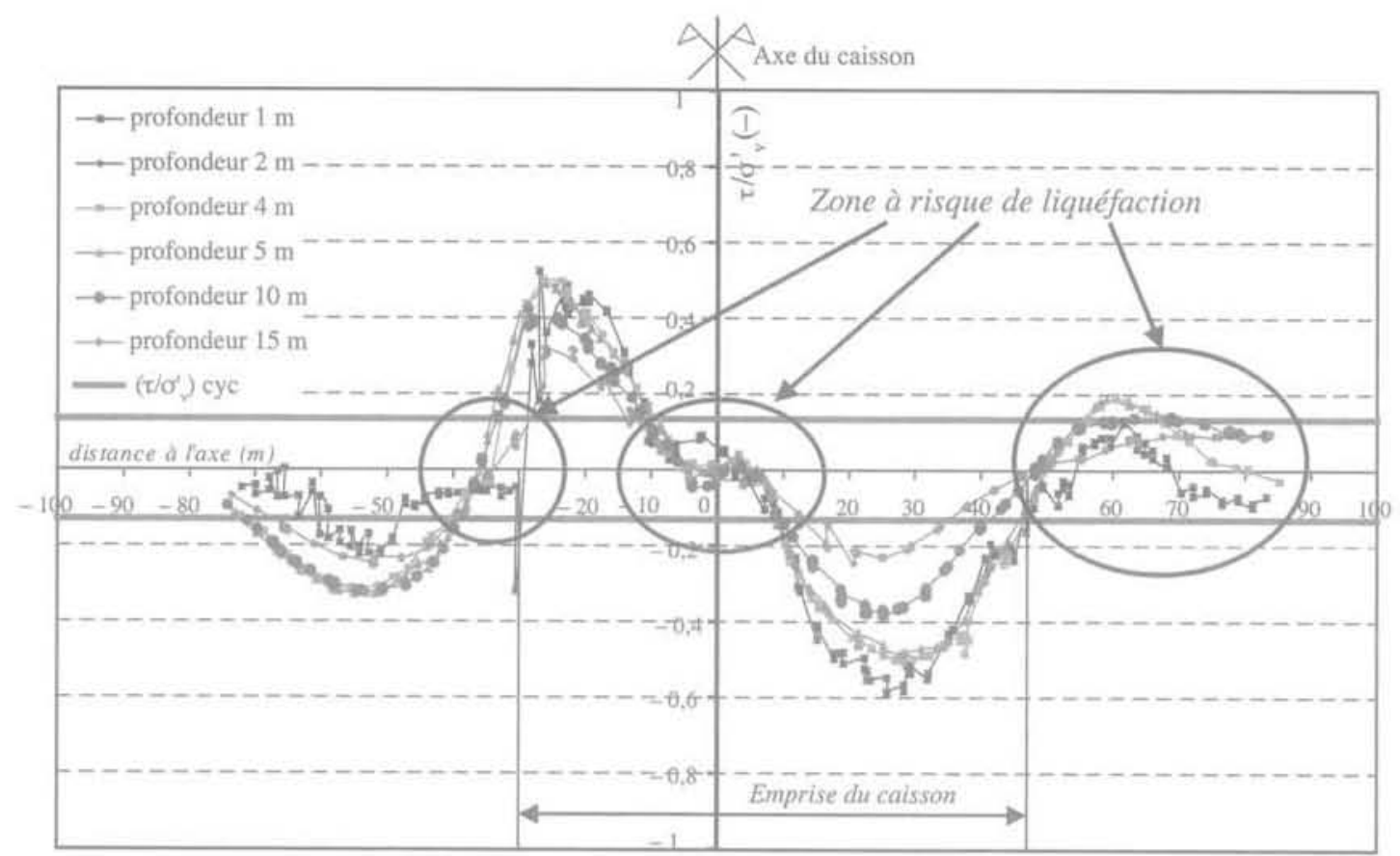

FG. 9 Ratio de contrainte de cisaillement sous l'ouvrage. Cyclic shear stress ratio under the caisson.

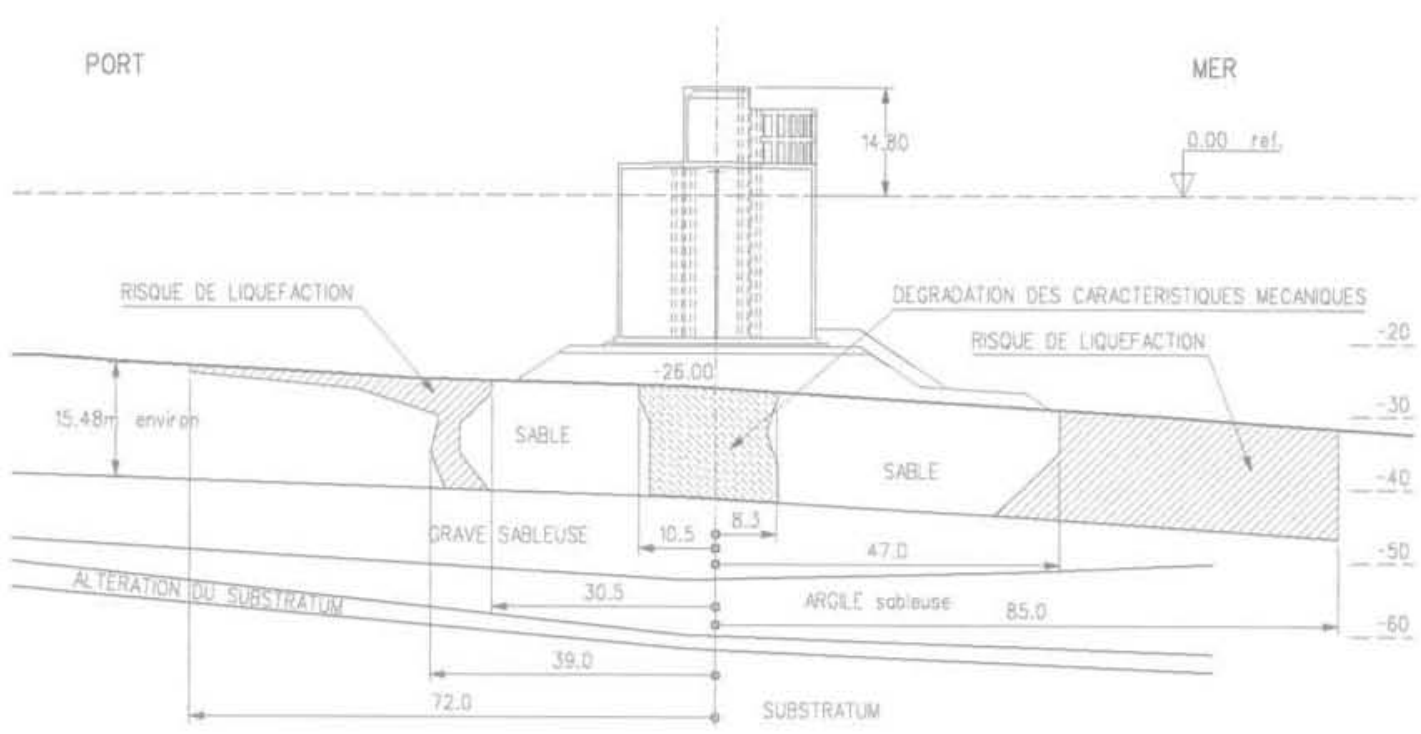

Port de Tanger-Méditerranée : digue principale. Coupe transversale vers le PM 1695.

FG. to Impact de l'aléa sismique sur le sol chargé par l'ouvrage. impact of seismic event on loaded ground.

Une fois tracées ces courbes à différentes profondeurs, il est facile de superposer deux droites horizontales figurant la valeur absolue du CSR (Cyclic Stress Ratio), lequel varie également en fonction de la profondeur, suivant la formule de Seed. On définit alors des zones où, à cause de la modification des contraintes due au chargement, la liquéfaction ne peut avoir lieu suivant le principe exposé au paragraphe précédent (seul le cas où la valeur de la contrainte de cisaillement est suffisamment élevée pour inverser la direction du cisaillement est susceptible de conduire à la liquéfaction). On constate que le rapport $\left(\tau / \sigma^{\prime}\right)$ préexistant est dans le cas présent augmenté jusqu'à des valeurs très nettement supérieures au CSR $(0,4$ à 0,5 à comparer à 0,13-0,15).
On en déduit alors un schéma de principe (Fig. 10) quj définit dans une coupe transversale l'étendue des zones susceptibles de liquéfaction. Ces zones sont situées en pied de digue et dans une bande centrée sur l'axe de l'ouvrage.

Il est intéressant de noter que dans l'axe de l'ouvrage, il existe effectivement une zone oủ le CSR est supérieur au rapport $\left(\tau / \sigma^{\prime}\right)$ préexistant et où la liquéfaction (totale ou partielle) est donc théoriquement possible, ce qu'ont effectivement montré certains essais de laboratoire. En réalité, dans les conditions réelles, compte tenu du confinement exercé latéralement et en surface, la liquéfaction serait vraisemblablement remplacée par un affaiblissement des caractéristiques mécaniques du sol, amenant par exemple des tassements (Zhang et al., 2002). 


\section{Étude de l'influence des phénomènes de liquéfaction sur la stabilité de la digue : calculs pseudo-statiques}

Dans cette partie de l'étude nous avons cherché à étudier la stabilité de la digue sous séisme avec liquéfaction, en analysant l'impact de grands glissements sur des surfaces de rupture passant dans les sables, la rupture étant potentiellement générée par un mécanisme consécutif à l'augmentation de pression interstitielle dans les terrains sous et à côté de l'ouvrage.

En effet, le CCTP stipulait : « La stabilité des ouvrages sera évaluée par des calculs pseudo-statiques tels que décrits dans l'AFPS (ouvrage poids déplaçable) prenant en compte la dégradation des caractéristiques mécaniques des zones liquéfiables, incluant des analyses de rupture circulaire (type Talren). On visera un coefficient de sécurité de 1 sous charges non pondérées en tenant compte du caractère non simultané du pic d'accélération et de l'apparition de la liquéfaction. »

Les calculs de stabilité pseudo-statiques usuels type AFPS, n'intégrant que la valeur de l'accélération, auraient privilégié l'influence du séisme court au détriment du séisme long et n'auraient pas été représentatifs.

Des calculs ont donc été faits avec le logiciel Talren (Fig. 11), en utilisant la possibilité qu'il offre de modifier localement le champ des pressions hydrostatiques à partir d'un maillage éléments finis fait sur Plaxis. L'influence de la liquéfaction est prise en compte par une augmentation de la valeur des pressions interstitielles, à rajouter à la valeur hydrostatique initiale dans le terrain (niveau de nappe $+1,00$ ).

Pour les zones en amont et à l'aval on a supposé une augmentation de pression interstitielle égale à $60 \%$ de la valeur de la contrainte verticale effective.
Dans la zone centrale sous le caisson nous avons considéré un incrément de pressions interstitielles $\Delta \mathrm{u}$ égal à $0,3 \sigma_{v^{\prime}}^{\prime}$

Dans les zones ou le risque de liquéfaction était le plus avéré, la prise en compte de ces hypothèses de pression interstitielle ramenait le coefficient de sécurité vis-à-vis de la rupture au grand glissement de 1,5 dans le cas statique aux alentours de 1,1 ou 1,2.

L'étude de la stabilité au glissement sous l'effet de l'augmentation des pressions interstitielles dans la zone aval et sous le centre de l'ouvrage conduisait à des coefficients de sécurité globalement supérieurs à 1,1, ce qui était acceptable vis-à-vis de la stabilité d'ensemble.

L'étude de stabilité montrait par ailleurs que l'augmentation de la pression hydrostatique dans la zone amont ne semblait pas avoir d'influence (ou très peu) sur la stabilité de la digue vis-à-vis du glissement le long de la surface de rupture concernée. Ceci dit, un basculement vers l'amont du caisson consécutif à un tassement différentiel entre l'amont et le centre de la structure n'était pas à exclure.

L'influence de la berme en pied de caisson a également été étudiée. Par rapport au cas statique équivalent, le coefficient de sécurité en cas de liquéfaction est ramené de 3,5 à 1,9. Enfin, on a également analysé la sécurité de la stabilité du caisson vis-à-vis d'une rupture due un défaut de portance du sol consécutive au phénomène de liquéfaction.

Les calculs de contrainte de rupture sous une fondation superficielle à partir de l'angle de frottement interne du matériau (Meyerhof) ont démontré que le risque de rupture du type portance sous un caisson de $28 \mathrm{~m} \times 28 \mathrm{~m}$ avec une contrainte de référence de $300 \mathrm{kPa}$ n'apparaissait que pour un angle de frottement résiduel de l'ordre de $19^{\circ}$. Or les essais de cisaillement statique à rupture post-cyclique n'ont jamais mis en évidence une valeur aussi faible de l'angle de frottement du matériau, tous les essais effectués donnant des valeurs de l'angle de frottement résiduel comprises entre 40 et $43^{\circ}$.

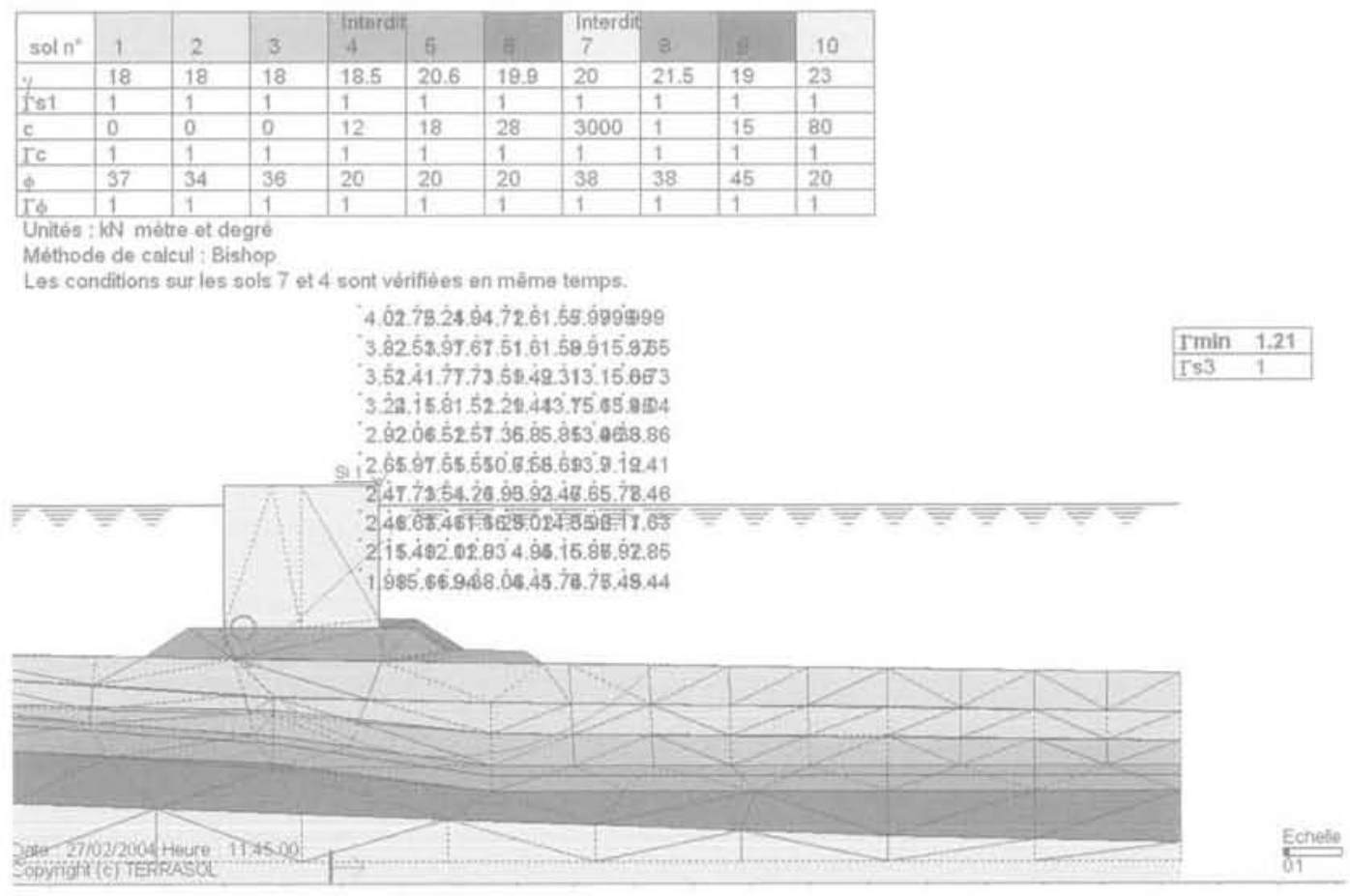

FIG, 11 Analyse de stabilité pseudo-statique.

Pseudo-static stability analysis. 


\section{9}

\section{Étude de l'influence des phénomènes de liquéfaction sur la stabilité de la digue : calculs dynamiques}

\section{1}

\section{Les raisons d'une telle étude}

A ce stade de l'étude, on pouvait estimer que l'action des sollicitation sismiques les plus défavorables se traduisait dans les zones les plus sensibles, d'une part, par un risque de liquéfaction partielle amenant une diminution des caractéristiques mécaniques des sols sous et autour de l'ouvrage et, d'autre part, par une augmentation significative de la pression interstitielle de part et d'autre des caissons. Le risque de voir les caissons et donc les superstructures de la digue affectés par des mouvements de basculement et de rotation, et par des tassements, a amené le maître d'ouvrage à demander au groupement d'entreprises de procéder à une analyse dynamique d'interaction sol-structure afin de vérifier les résultats obtenus par les analyses pseudo-statiques et également d'essayer de quantifier l'importance des désordres pouvant affecter l'ouvrage, sachant que le risque de ruine de l'ouvrage était a priori exclu.

Cette étude dynamique a comporté deux étapes : - tout d'abord la recherche et le choix de signaux sismiques (accélérogrammes et spectres) compatibles avec les données sismiques de base définies au $\$ 4$; - ensuite l'utilisation de ces signaux dans un logiciel permettant de modéliser l'interaction sol-structure.

\section{2}

\section{Recherche de signaux sismiques représentatifs}

La recherche de signaux sismiques ad hoc a été confiée par le groupement à Geoter.

Les données sismiques initiales (\$ 4) ne comportant pas de spectres de référence, Geoter a donc calculé en préalable des spectres de réponse élastique pour le site (spectres cibles) en adoptant les lois d'atténuation utilisées dans l'étude de l'université Mohammed V. Ensuite, il lui a fallu sélectionner dans les bases de données accélérométriques (USGS 1996; Ambraseys et al., 2000) des enregistrements correspondant à des séismes de caractéristiques proches des séismes de référence, en calant les composantes horizontales à l'accélération de dimensionnement et en vérifiant le contenu spectral de l'accélérogramme par comparaison des spectres de réponse élastique de ses composantes au spectre cible résultant de l'application des lois d'atténuation.

GEOTER a sélectionné les séismes suivants :

\begin{tabular}{l|l|c|c} 
& \multicolumn{1}{|c|}{ Nom } & Date & Durée (s) \\
\hline Séismes proches & Friuli (Italie) & $16 / 08 / 1977$ & 16 \\
\hline & Izmir (Turquie) & $09 / 12 / 1977$ & 5 \\
\hline & Lazio Abruzzo (Italie) & $11 / 05 / 1984$ & 26 \\
\hline & Umbria (Italie) & $29 / 04 / 1984$ & 26 \\
\hline Séismes lointains & Kocaeli A (Turquie) & $17 / 08 / 1999$ & 106 \\
\hline & Kocaeli B (Turquie) & $17 / 08 / 1999$ & 140 \\
\hline & Kocaeli C (Turquie) & $17 / 08 / 1999$ & 25 \\
\hline
\end{tabular}

Les signaux fournis comprenaient à chaque fois deux composantes horizontales orthogonales et une composante verticale.

\section{3}

\section{Modélisation de l'interaction dynamique sol-structure}

Cette étude a été confiée à Terrasol qui a utilisé pour ce faire le code Plaxis (module dynamique). Deux profils ont été étudiés, l'un correspondant à la zone jugée la plus critique en terme de risque de liquéfaction, l'autre à des terrains représentatifs de la zone courante.

Ce type d'étude pose un certain nombre de problèmes qu'il a fallu résoudre.

\section{3 .1}

\section{Choix du signal}

Plaxis prend en compte un signal temporel pour le séisme qui est positionné à la base du modèle, au niveau du substratum. Le logiciel développe analytiquement l'amplification, l'amortissement et la duplication des ondes aux interfaces géologiques. Le signal est représenté par une série de points par pas de temps régulier représentant soit le déplacement, soit la vitesse, soit l'accélération du substratum en fonction du temps.

Plaxis n'accepte qu'un seul signal. La prise en compte simultanée des composantes horizontale et verticale du séisme est réalisée par un coefficient multiplicateur variable sur le signal. Pour l'étude il a été décidé de prendre en compte simultanément la composante horizontale de l'accélération et comme composante verticale, une fraction de $30 \%$ de la composante horizontale.

Le choix des signaux parmi ceux proposés par Geoter s'est fait en fonction de leur contenu fréquentiel, de la durée du séisme et sur l'allure générale du signal. C'est ainsi qu'ont été sélectionnés comme les plus représentatifs les accélérogrammes suivants : séisme de Kocaeli (Turquie) du 17/08/1999 comme référence pour le séisme de source lointaine, et séisme d'Ombrie du 29/04/1984 comme référence pour le séisme de source proche.

Les deux accélérogrammes sont représentés respectivement sur les figures 12 et 13. Le signal du séisme de Kocaeli A avait l'avantage d'être de longue durée (plus de 100 secondes) et présentait donc un intérêt certain pour l'étude des phénomènes relatifs à la liquéfaction car il impliquait un nombre de cycles représentatif.

Le contenu fréquentiel des séismes lointains est plus riche dans les basses fréquences (entre 0,7 à $3 \mathrm{~Hz}$ ) que celui des séismes proches, plus développé dans les hautes fréquences ( 3 à $10 \mathrm{~Hz}$ ). Il est alors plus préjudiciable pour la digue en caisson dont la fréquence propre est proche de $1 \mathrm{~Hz}$, malgré une accélération maximale 2,5 fois plus faible.

\section{$9 \sqrt{352}$}

\section{Prise en compte de l'effet de l'eau}

L'approche utilisée est celle des masses ajoutées de Newmark (1971). Pour une structure longitudinale (que l'on peut assimiler à un prisme rigide sur un support flexible) se déplaçant dans un fluide perpendiculaire- 


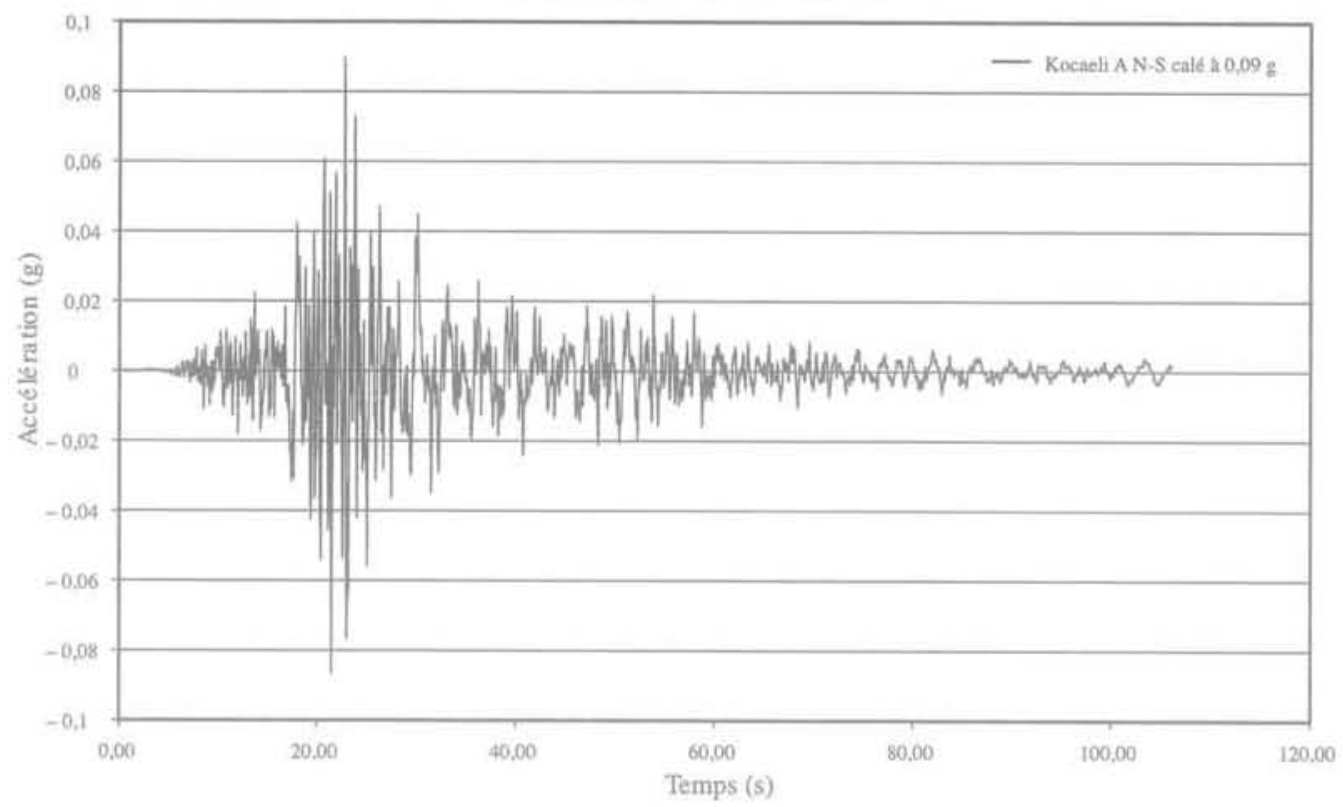

EiG, 12 Séisme long jugé représentatif.

Typical long duration seismic event.

Accélérogramme Umbria E-W

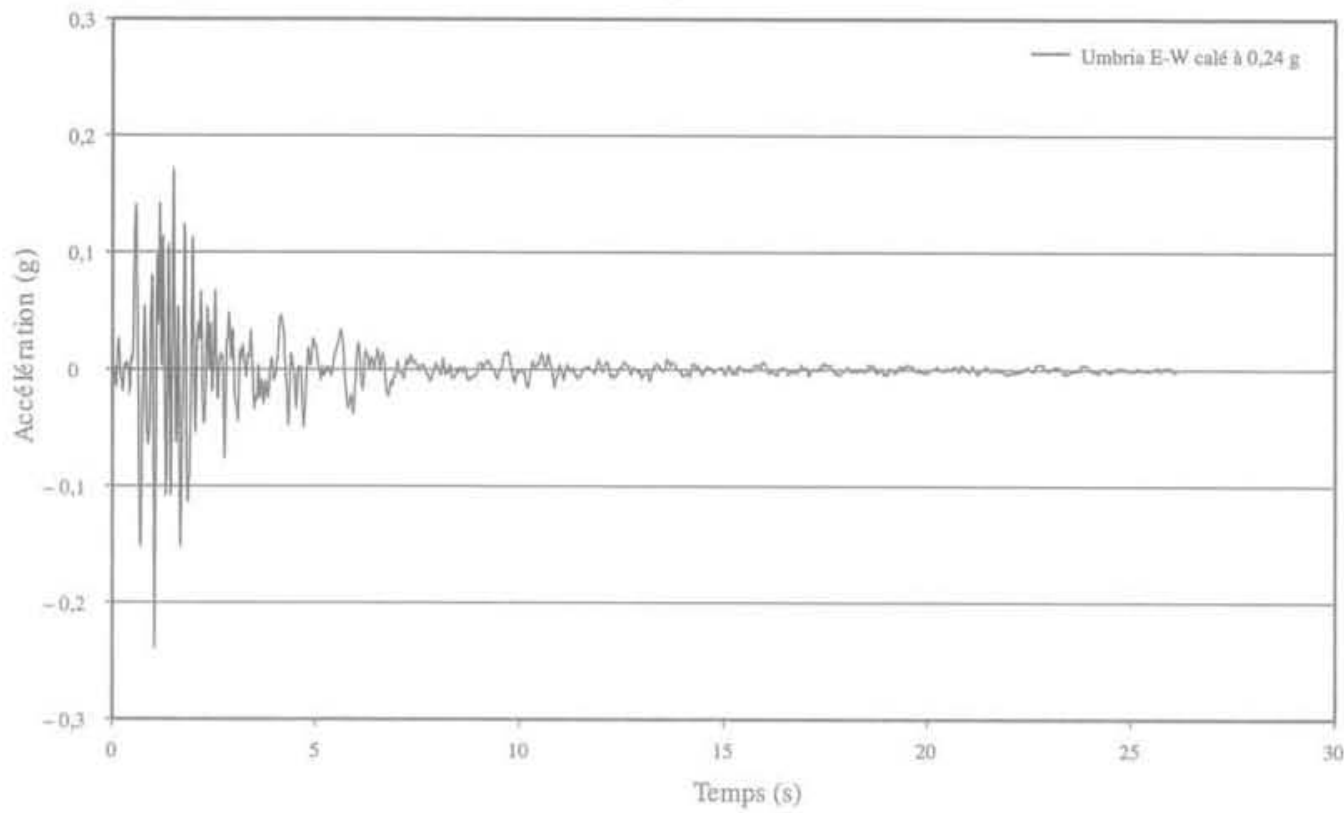

FG. 13 Séisme court jugé représentatif.

Typical short duration seismic event.

ment à son axe longitudinal, la masse ajoutée est évaluée par la masse d'un cylindre de liquide ayant un diamètre égal à la hauteur de la structure immergée dans l'eau.

Ainsi pour un caisson de hauteur $24 \mathrm{~m}$ immergé dans l'eau sur une hauteur de $21 \mathrm{~m}$ la masse ajoutée correspond à celle d'un volume $\Pi \times 21^{2} / 4=346 \mathrm{~m}^{3} / \mathrm{m}$ soit $353 \mathrm{t} / \mathrm{m}$ réparties sur le voile central du caisson.

En pratique, l'effet de ces masses ajoutées est de diminuer la fréquence de résonance du système et de limiter les déformations du caisson. A contrario, elles augmentent la masse totale du caisson et donc les tassements sous le caisson. Il a donc été décidé d'étudier comparativement le comportement du caisson avec et sans masses ajoutées.

\subsubsection{3}

\section{Paramètres géotechniques}

Les nombreux essais en laboratoire (essais de cisaillement cycliques, colonne résonnante) ont permis une modélisation réaliste du comportement des sables. Ceux-ci ont été modélisés avec un modèle élastique non linéaire de type HSM (hardening soil model selon le manuel d'utilisation de Plaxis).

Le modèle HSM définit une relation non linéaire entre le déviateur q et la déformation $\varepsilon$ :

$$
\mathrm{q}=2^{*} \mathrm{E}_{50}{ }^{\star} \varepsilon /\left(1+2^{*} \mathrm{E}_{50} / \mathrm{q}_{\mathrm{a}}{ }^{*} \varepsilon\right)
$$


avec $\mathrm{q}_{\mathrm{a}}=\mathrm{q}_{\mathrm{f}} / \mathrm{r}_{\mathrm{f}}, \mathrm{q}_{\mathrm{f}}$ étant le déviateur à rupture mesuré par exemple lors d'essais triaxiaux, et $\mathrm{q}_{\mathrm{a}}$ la valeur asymptotique à l'infini (on prend souvent la valeur de 0,9 pour $r$ ).

Pour un sol répondant au critère de rupture de Mohr-Coulomb la valeur de $\mathrm{q}_{\mathrm{f}}$ vaut :

$$
q_{f}=\left(K_{p}-1\right)^{*} \sigma_{3}^{\prime}+2 c^{*} \cos \varphi /(1-\sin \varphi)
$$

Pour un sol de type HSM, trois modules caractéristiques sont à définir : $\mathrm{E}_{\max }$ (module tangent à l'origine), $E_{50}$ et $E_{u r}$ (décharge/recharge). Le module $E_{50}$ se déduit par exemple à partir de résultats d'essais triaxiaux donnant la valeur du demi-déviateur à rupture et la déformation correspondante.

Le module $\mathrm{E}_{\mathrm{ur}}$ est pris (à défaut d'informations spécifiques issues de résultats d'essais) par défaut comme $3 \times \mathrm{E}_{50^{\circ}}$

La figure 14 représente graphiquement les différents paramètres en question.

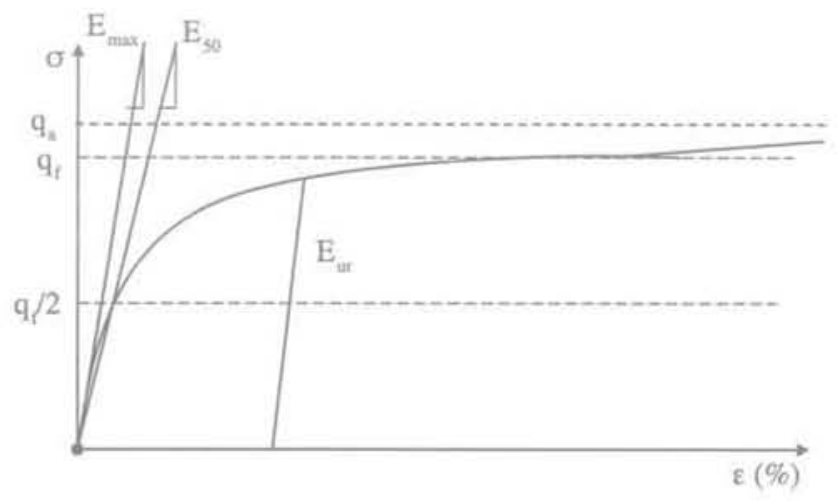

FiG. 14 Les paramètres du modèle HSM Plaxis. HSM Plaxis parameters.

Les valeurs de module utilisées dans le programme PLAXIS correspondent à la valeur du module $E_{50}$ ret ramené à la pression de référence $\mathrm{p}_{\text {ref }}=100 \mathrm{kPa}$ et défini par la formule:

$$
E_{50}=E_{50}{ }^{r+*}\left[\left(c^{*} \operatorname{cotg} \varphi+\sigma^{\prime} 3\right) /\left(c^{*} \operatorname{cotg} \varphi+p_{\text {ref }}\right)\right]^{m}
$$
$m$ variant entre 0,5 pour des sables lâches à moyennement consolidés et 0,65 pour des sables denses.
Le module tangent en un point quelconque de la courbe $E=d q / d \varepsilon$ vaut alors par dérivation de la formule ci-dessus :

$$
E=2^{*} E_{50} /\left(1+2^{*} E_{50} / q_{a}{ }^{\star} \varepsilon^{2}\right.
$$

Pour $\varepsilon=0$ on a $\mathrm{E}=2 \mathrm{E}_{50}=\mathrm{E}_{\max }$ module tangent à l'origine,

Sachant que $\mathrm{G}=\mathrm{E} /\left(2^{*}(1+v)\right)$ et que la distorsion $\gamma$ est égale à $(1+v)^{*} \varepsilon$, il vient :

$\mathrm{G}=2^{\star} \mathrm{G}_{50 \mathrm{j}} /\left[1+4^{*} \mathrm{G}_{50} / \mathrm{q}_{0}{ }^{*} \gamma\right]^{2}$ avec $\mathrm{G}_{50}=\mathrm{E}_{50} /(2(1+v))(5)$

Pour $\gamma=0$ on a $G=G_{\max }=2 G_{50}$ et la formule précédente s'écrit:

$$
\mathrm{G} / \mathrm{G}_{\max }=1 /\left[1+4^{*} \mathrm{G}_{50} / \mathrm{q}_{\mathrm{a}}{ }^{*} \gamma\right]^{2}
$$

Ce qui se traduit par une courbe représentée dans la figure 15.

Les résultats des différents essais ont révélé un coefficient multiplicateur entre paramètres « statiques » et " dynamiques ») compris entre 5 et 8.

Il a été choisi d'appliquer un coefficient multiplicateur de 6 entre modules statiques et modules dynamiques dans les sols relativement lâches.

Pour les sols plus raides (graves sableuses, substratum argileux) et les éléments de construction (ballast, carapace), un coefficient multiplicateur plus faible (4) a été appliqué, afin de ne pas raidir excessivement dans le calcul dynamique la structure mise en place.

\section{$9.3 \times 4$ \\ Résultats des calculs : déformations, surpressions interstitielles}

Les déformations irréversibles en fin de séisme apparaissent comme relativement limitées car les déplacements horizontaux du caisson et les tassements observés en fin de séisme n'excédent pas $5 \mathrm{~cm}$ (Fig. 16), Ils s'élèvent à $7 \mathrm{~cm}$ au maximum pendant le séisme long et sont deux fois moins importants avec le séisme court. Des déformations irréversibles de consolidation post-sismique ont également été mises en évidence mais elles sont limitées à des valeurs de l'ordre de 10 à $15 \mathrm{~mm}$.

Le séisme lointain génère plus de distorsions $(\gamma=1,2 \%)$ que le séisme court $(0,3$ à $0,6 \%)$.

Tanger SC $1083 \mathrm{~m}$

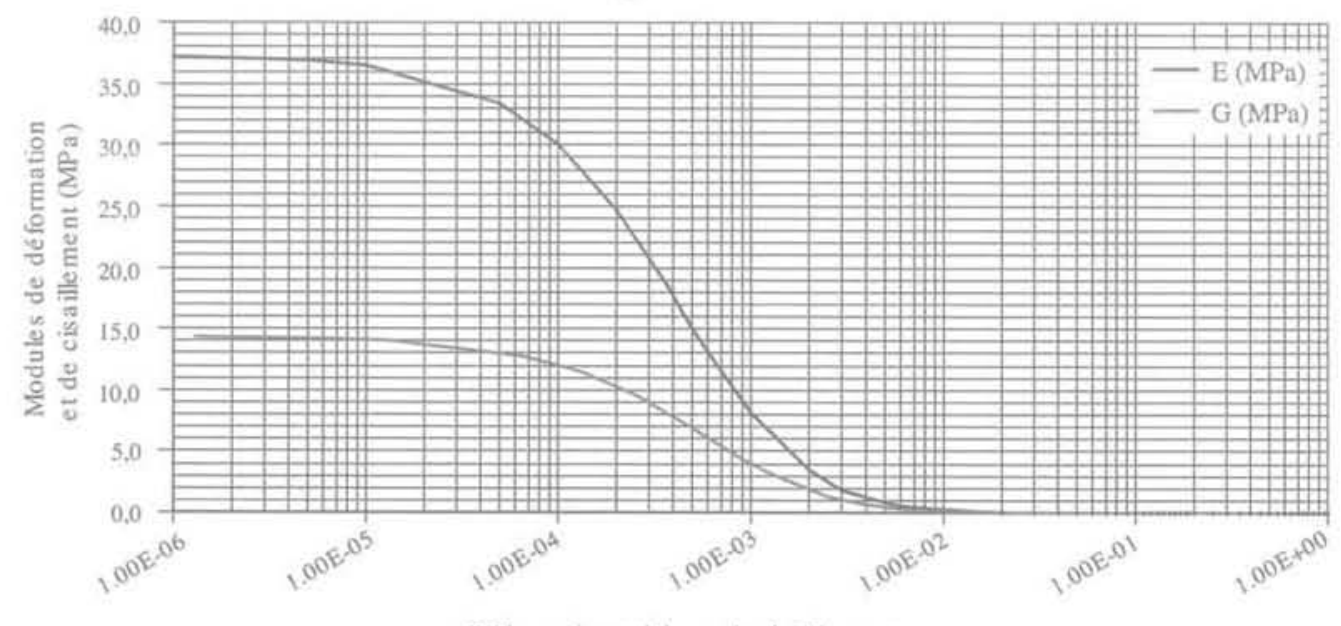

Déformation axiale ou de cisaillement 

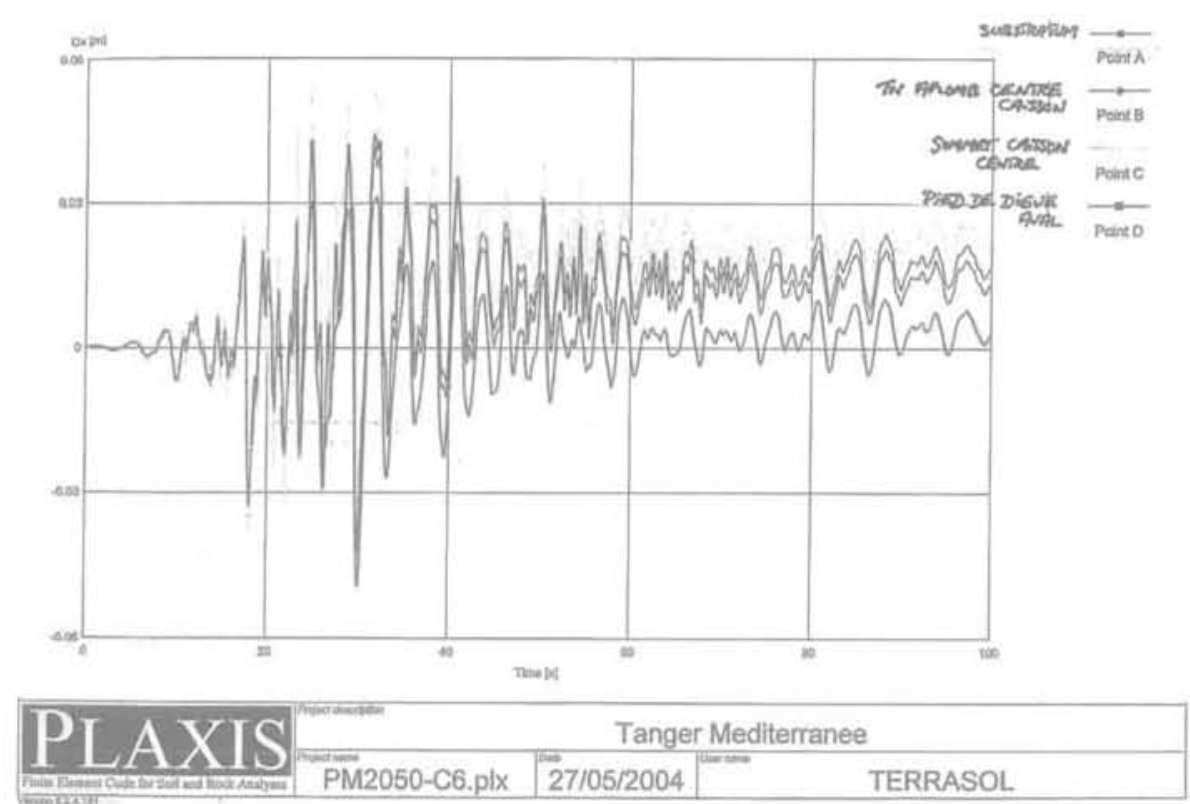

16.16 Évolution des déplacements horizontaux pendant le séisme. Horizontal displacements during seismic event.

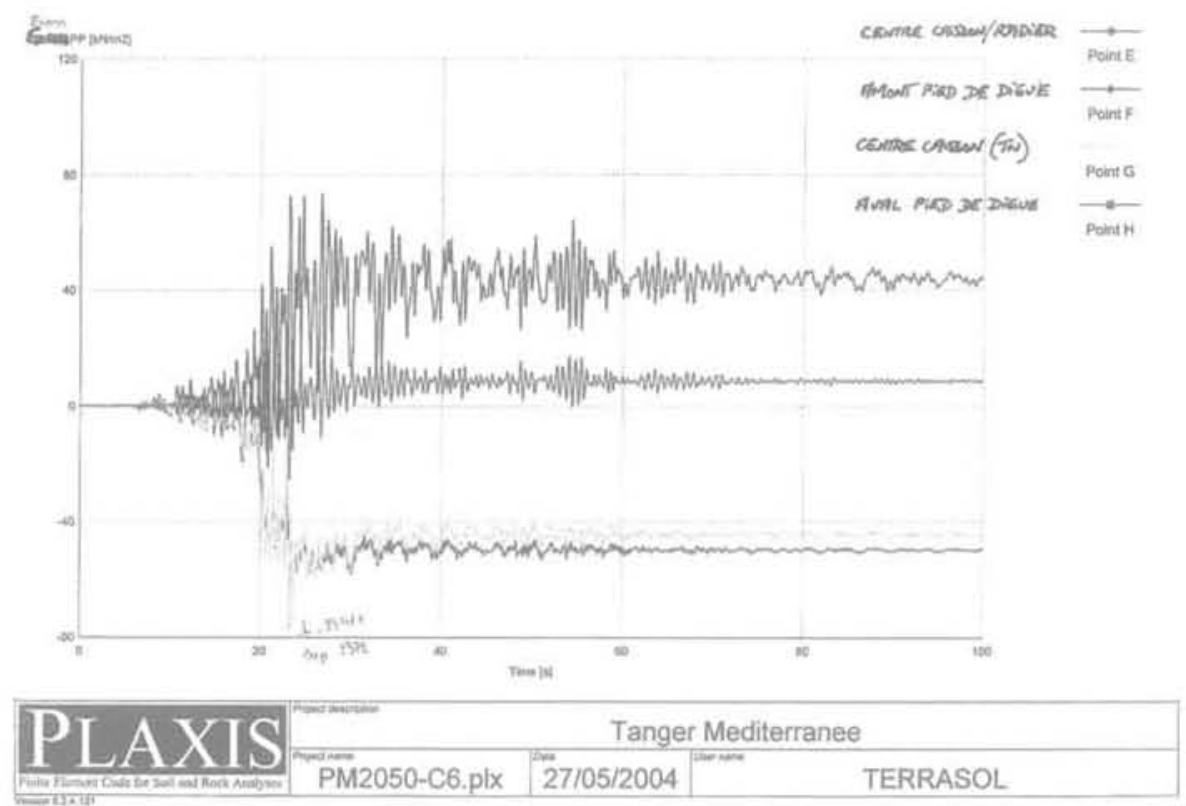

FG, 17 Évolution des pressions interstitielles pendant le séisme. Pore pressure development during seismic event.

Les surpressions interstitielles sont essentiellement générées dans les couches d'argile sous-jacentes, là où I'on observe les distorsions les plus importantes. A la verticale du milieu du caisson on observe des valeurs de surpressions interstitielles de l'ordre de $50 \mathrm{kPa}$ dans les sables silteux sous le matériau de remblai, ce qui corrobore les hypothèses faites précédemment d’une augmentation de 30 à $60 \%$ de la pression hydrostatique (Fig. 17).

\subsection{5}

\section{Amplification/atténuation du signal sismique}

L'étude dynamique d'interaction sol-structure a permis également de donner des informations sur ce point. En effet, les accélérations de projet étaient défi- nies au toit du substratum rocheux ; il se posait donc la question de l'évolution de ces accélérations dans les terrains meubles sus-jacents.

Les diagrammes d'accélération montraient dans trois cas sur quatre ( 2 profils $\times 2$ types de séisme) une atténuation de l'accélération entre le niveau du substratum et la surface du sol. Par contre, dans un cas de figure correspondant à des terrains jugés plutôt raides et au séisme long, l'accélération passait de 0,09 g au niveau du substratum à $0,15 \mathrm{~g}$ au niveau de la surface (Fig. 18). C'est d'ailleurs dans cette configuration (terrains plutôt raides et séisme long) que l'on obtenait les déplacements les plus importants. A contrario, la zone la plus sensible à la liquéfaction montrait systématiquement une atténuation de l'accélération entre le substratum et la surface quel que soit le type de séisme. 


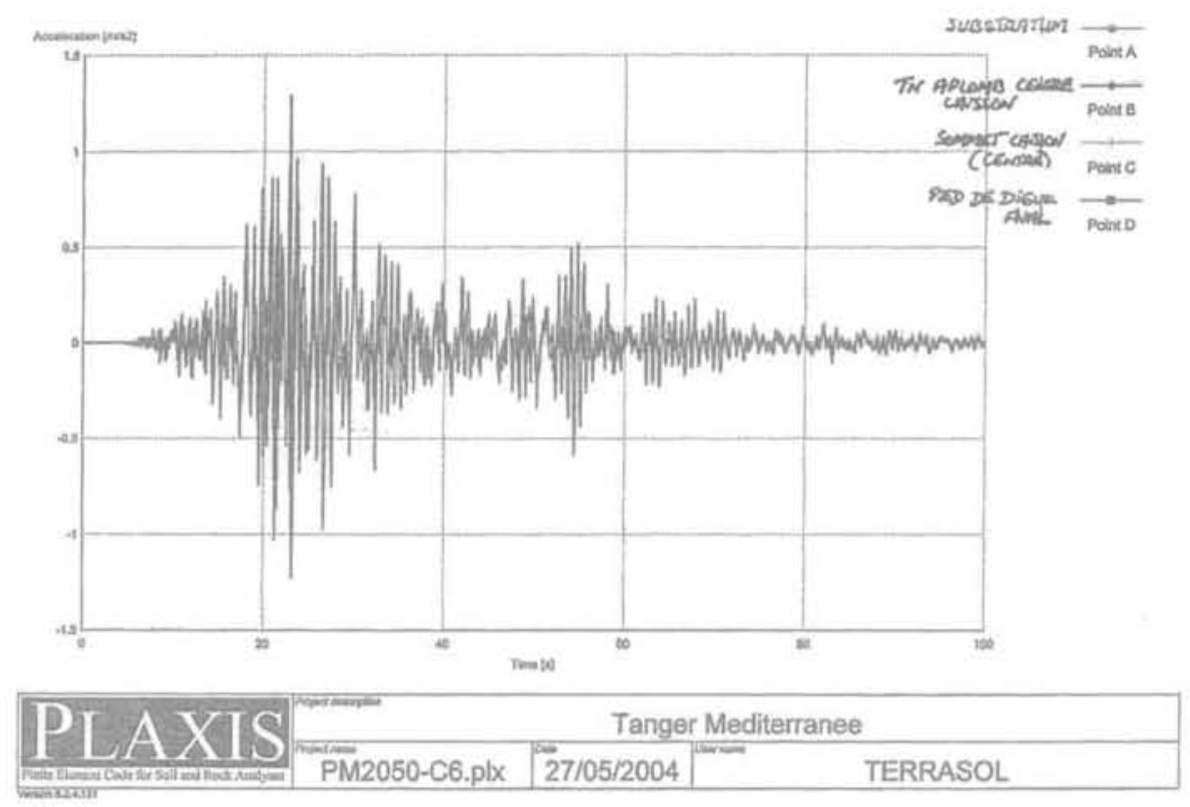

F19.18 Accélérations observées pendant le séisme.

Accelerations during seismic event.

\section{0}

\section{Conclusion}

Cette étude a permis une analyse exhaustive du risque de liquéfaction des terrains sous-jacents à la digue en caissons du nouveau port de Tanger-Méditerranée. Elle s'est en effet appuyée sur plusieurs phases successives :

- essais in situ (CPT et SPT) ;

- essais de laboratoire ;

- étude de l'influence du chargement apporté par les caissons ;

- analyse de stabilité pseudo-statique avec prise en compte de l'accroissement de pression interstitielle ; - étude dynamique d'interaction sol-structure.

Ces différentes phases ont permis d'éclairer les résultats ambigus, voire contradictoires, obtenus dans la phase initiale des essais in situ, phase à laquelle les études de liquéfaction des sols sont trop souvent réduites.

Nous en avons retenu les enseignements suivants :

1) Les essais in situ donnent des résultats apparemment contradictoires, les essais CPT donnant à penser qu'il y a effectivement risque de liquéfaction alors que les SPT ne mettent pas en évidence ce phénomène. A la lumière des analyses ultérieures il s'avère que les CPT donnent une perception plus juste, quoique conservatrice, de la situation. L'analyse par les essais in situ avec les outils actuels (recommandations NCEER) permet par contre de mettre clairement en évidence l'influence de la magnitude en complément de l'accélération.

2) Les essais en laboratoire ont mis en évidence que le risque de liquéfaction est réel avec certains sols concernés, en particulier dans la zone de dépôt de matériaux alluvionnaires apportés par l'oued R'Mel mais pour un niveau de sollicitations sismiques supérieur de $50 \%$ aux hypothèses envisagées $(0,13 / 0,15 \mathrm{~g}$ au lieu de $0,093 \mathrm{~g}$ ), le phénomène ayant été simulé plu- sieurs fois dans des essais de cisaillement cyclique. Pour les fortes densités relatives la liquéfaction n'est pas atteinte, même pour des accélérations de l'ordre de $0,15 \mathrm{~g}$. Les essais de laboratoire apportent donc un éclairage indispensable dans le cas relativement courant où l'intensité des sollicitations sismiques n'est pas manifestement complètement destructrice.

3) Pour le niveau de sollicitations sismiques de projet et non atténuées, les phénomènes auxquels on peut s'attendre correspondent à une augmentation relative partielle des pressions interstitielles de l'ordre de 30 à $60 \%$ accompagnée d'une diminution sensible des caractéristiques de déformation (modules). Il s'agit d'un phénomène que l'on pourrait appeler liquéfaction partielle, mais non liquéfaction au sens strict du terme. Les risques liés à cette augmentation des pressions interstitielles est en partie, mais en partie seulement, annihilé par l'influence du chargement apporté à l'ouvrage, puisque l'analyse de l'effet du chargement sur la répartition des contraintes montre qu'il subsiste une bande centrale et des bandes latérales où le chargement n'induit pas d'effet d'augmentation de la contrainte de cisaillement statique, ce qui est un facteur déterminant vis-à-vis de la sensibilité à la liquéfaction.

4) Seules des analyses élaborées permettent alors d'estimer l'influence de cette dégradation des caractéristiques de déformation des terrains ; en particulier des études de stabilité pseudo-statiques basées sur la seule valeur de l'accélération auraient amené à des conclusions disproportionnées, alors que l'étude de stabilité au glissement montre qu'avec l'augmentation de la pression interstitielle envisagée dans les zones concernées et sans atténuation, les coefficients de sécurité obtenus sous liquéfaction partielle restent supérieurs à 1,1 ce qui exclut à priori la nécessité d'un traitement de terrain.

5) Compte tenu de la diminution des caractéristiques de déformabilité des matériaux, observée dans les essais de laboratoire, la liquéfaction partielle est susceptible de générer des déplacements (glissements et 
rotations) non négligeables, leur quantification ne pouvant se faire qu'au travers d'une analyse détaillée d'interaction sol-structure sous sollicitations dynamiques. Ce type d'étude, lourde au demeurant, requiert une démarche rigoureuse et vigilante dans le choix des paramètres et des hypothèses de calculs (choix des signaux, prise en compte de l'effet de l'eau, lois de com- portement élaborées). Ce type d'analyse permet également d'apporter des réponses précises quant à l'effet d'amplification ou d'atténuation de l'accélération par les terrains sus-jacents au substratum. Dans le cas présent, pour ce type d'ouvrage, il a été observé que les terrains les plus sensibles au risque de liquéfaction atténuaient l'accélération au lieu de l'amplifier.

\section{$\overline{\text { Bibliographie }}$}

Andersen K.H., Berre T. - Behavior of a dense sand under monotonic and cyclic loading. ECSMGE Geotechnical Engineering for Transportation Infrastructure, vol, 2., Balkema, 1999, p. 667-676.

Martin G.R. Finn W.D.L. Seed B. B - Fundamentals of liquefaction under cyclic loading. Journal of Geotechnical Engineering Division, ASCE, vol. 101, n GT5, 1975, p. 423-438.

Newmark N.M., Rosenblueth E. - Fundamentals of earthquake engineering. Prentice Hall Inc., 1971.

Pecker A. - Dynamique des sols. Presses des Ponts et Chaussées, 1984.

PLAXIS 2D V8 Code de calcul géotechnique aux éléments finis. Manuel d'utilisation, (Material model and dynamic model manuals), Balkema, 2002.

Recommandations AFPS 90 pour la rédaction de règles relatives aux ouvrages et installations à réaliser dans les régions sujettes aux séismes. Presses des Ponts et Chaussées, 1990

Rollins K.M. - The influence of buildings on potential liquefaction damage. $\mathrm{PhD}$ dissertation, University of California, Berkeley, 1987

Seed R.B., Idriss I.M. - Simplified procedure for evaluating soil liquefaction potential. Journal of the Soil Mechanics and Foundations Division, ASCE, SM9. 1971, p, 1249-1273.

Seed R.B. et al. - Recent advances in soil liquefaction engineering: a unified and consistent framework. $26^{\text {th }}$ Annual ASCE Los Angeles Geotechnical Spring Seminar, Report n EERC, 2003.

Vaid Y.P., Sivathayalan S. - Fundamental factors affecting liquefaction susceptibility of sands. Canadian Geotechnical Journal n 37,2000 , p. 592-606.
Vaid Y.P. Stedman J.D, Sivathayalan S, Confining stress and static shear effects in cyclic liquefaction. Canadian Geotechnical Journal $n^{\circ} 38,2001$, p. 580-591.

Youd L.T., Idriss I.M. et al, - « Liquefaction resistance of soils x, Summary Report from the 1996 NCEER and 1998 NCEER/NSF Workshops on evaluation of liquefaction resistance of soils. Journal of Geotechnical and Geoenvironmental engineering, $\mathrm{ASCE}$, vol. $127, n^{\circ} 4$ 2001, p. 297-313

Zhang G., Robertson P.K., Brachman R.W.JEstimating liquefaction-induced ground settlements from CPT for level ground. Canadian Geotechnical Journal n ${ }^{\circ} 39,2002$, p. 1168-1180, 2002. 